\title{
2020 Undergraduate Big Data Challenge: Personal and Public Health Decisions in a New Open Data Reality
} Sponsored by: Roche, SAS, Canadian Science Publishing, Digital Science, Altmetric, and Overleaf

The STEM Fellowship Big Data Challenge for undergraduate students is an inquiry-driven experiential learning program that affords students an opportunity to develop and strengthen their problem-solving and critical thinking skills while gaining familiarity with the fundamentals of data science (an important skill for the digital age). The students are presented with a number of well-designed workshops that introduces and enhances their knowledge on a broad range of data analytics tools and programming languages which are useful for uncovering hidden patterns, trends in structured and unstructured data. Some of the tools and programming languages the students learnt and used includes Python, R, SAS, Overleaf, and Machine learning.

The programming languages and data analytical tools introduced to the students are considered essential and fundamental to perform big data exploration. Given the unprecedented situation the world was challenged with this year with the COVID-19 pandemic, many of the projects undertaken by the student teams explored how COVID-19 may have impacted the different facets of our lives and society. Some of the scope of the work examined includes: economic impacts, microbiology, efficacy of PPE regulations, among others. For all the variations between project themes, it remains that all submissions are of incredibly high quality. Every paper is demonstrative of immense creativity and high potential on the respective team's part.

On behalf of STEM Fellowship, I would like to extend my heartfelt congratulations to all students who participated in the challenge, and I wish them all the best for their future endeavours in research and data science. It has been a privilege for us to witness the analytical capabilities of the next generation of students firsthand, and I am certain all entrants will continue to demonstrate excellence in their respective research careers.

\section{Anish Verma}

Chief Data Officer
Dr. Sacha Noukhovitch

Founder, President and Editor-in-Chief

\section{Disclaimer}

These abstracts are provided for all student teams that have submitted project reports by June 1, 2020. The STEM Fellowship Journal editorial board has made every effort to ensure proof and English editing of these abstracts in a limited amount of time, and neither organization as a whole or any of its volunteer members can be held accountable for inaccuracies that may have occurred in the abstract publication. Abstracts are published in alphabetical order per school names of the participating teams. 


\section{Analysis of Comorbidity Rate of COVID-19 and the Implications of Cancelling Cardiovascular Surgeries Due to COVID-19}

\author{
Ansari A., Irfan J., Rizvi S., and Fatima M. \\ University of Toronto Mississauga, McMaster University
}

This paper aims to determine the effects of chronic diseases on those suffering with COVID-19 by analyzing mortality and clinical characteristics in COVID-19 patients, as well as the impact of cancelling surgeries due to COVID-19. The key target of analysis from the datasets is the difference between clinical characteristics in COVID-19 patients with and without comorbidities, and identifying the effects of cancelled surgeries for chronic diseases as a result of COVID-19. This was analyzed to support that the number of cancelled surgeries may be detrimental, because chronic illnesses, particularly cardiovascular diseases, may be comorbid with COVID-19. Open access datasets - related to differences in clinical symptoms due to comorbidity between chronic illnesses and COVID-19 in China, number of deaths caused by COVID-19 and other leading causes of deaths in Canada and potential surgery cancellations in Canada - were analyzed using excel to create graphs for easier interpretation of data trends. The results illustrated that the mortality rate and severity of symptoms is higher in individuals who have two or more chronic diseases along with COVID-19 symptoms. Furthermore, data suggests that cardiovascular diseases are one of the leading causes of death in Ontario. The data also highlights that about 10,000 outpatient cardiovascular surgeries may be cancelled due to COVID-19. The key finding of this paper is that the number of panned cardiovascular surgeries should not be cancelled as both the mortality rate of cardiovascular diseases alone and along with COVID-19 symptoms is significantly high. Due to COVID-19, the healthcare system might be facing a risk in admitting high numbers of patients to stay in hospitals and many inpatient surgeries will be at risk of getting cancelled which is understandable. However, hospitals can give priority to outpatient surgeries because it is not necessary for the patients to stay in hospital post outpatient surgery.

Mentor: Omar Shafiq

Keywords: Cardiovascular diseases, Comorbidity, Coronavirus, Surgeries, Surgical cancellations

\section{A Convolutional Neural Network Approach for Skin Cancer Detection}

\author{
Andy Dai, Yi Zhou Tang, Kevin Zhu, and Nicolaus \\ Wong
}

Western University

Skin cancer is one of the most common cancers worldwide, and the incidences of skin tumours are slowly increasing globally. While most cases are benign, malignant cases are fatal if the diagnosis is overdue. Expert dermatologists and doctors can accurately identify malignant cancers in their early stages. However, invasive and intensive screening methods are used to diagnose a patient, which increases risks of infections, bleeding, and scarring for the patient. In addition to an increased risk, the hospital bears additional cost and time to complete the screenings. The aim of this study was to develop an automated diagnosis system that would improve the accuracy of cancer detection. Our method utilized the latest novel advancements in the field of Convolutional Neural Networks (CNN) to detect and assess a set of representative features for skin cancer. Our aim was to create a system that will diagnose patients faster than the traditional methods, be non-invasive, and significantly reduce risks placed on patients. The system provides greater accuracy in skin cancer detection and makes this application an efficient and dependable approach for dermatologists.

\section{Mentor: Rakhi Konar}

Keywords: Melanoma, Skin Cancer Detection, Convolutional Neural Network, Image Classification, Deep Learning

\section{Identifying COVID-19 Instagram Behaviour Patterns via a Novel Network Analysis Pipeline}

\section{Arthur Boschet, Vivian Chia-Jou Lee, Brenda Shen, and William Zhang}

\section{McGill University, Montreal, Canada}

Since its inception, Instagram has become one of the most used social media platforms in the world. The volume of information generated by Instagram users make it an excellent data mine to study human behavior. Recently, researchers have turned to Twitter data to observe the flow 
of (mis)information surrounding the COVID-19 pandemic. However, little to no publication has been done on coronavirus-related behaviours on Instagram. To address this knowledge gap, an extensive analysis of COVID-19-related data from Instagram was conducted. Hashtags obtained from 5,300 global Instagram posts collected between January 5, 2020 and March 20, 2020 were processed using a novel, highly extensible Social Network Analysis pipeline. This pipeline utilizes eigenvector centrality, weight cluster indexing and several other metrics. The analysis partitioned the hashtag data into clusters, three of which were studied to produce three key findings indicative of public behaviour. It was concluded that contributions to the mental health cluster consisted mainly of Instagram Business Profiles. Next, it was found that a niche cluster containing conspiracyrelated content was primarily created by genuine Instagram users. Lastly, hashtags related to comedy and memes were classified by a supervised machine learning approach to be bot-generated content. Based on these findings, this study can suggest promising directions for future investigations. Toward public health authorities, it is recommended that large-scale behavioral analysis of bots and their effects on COVID-19 information be performed. The conclusions drawn from this study include a need for more research that focuses on the intersectionality of mental health and COVID-19, as well as the rise of conspiracy theorists amidst this pandemic.

\section{Mentor: Randa Higazy}

Keywords: social network analysis, machine learning, eigenvector centrality, Instagram, mental health

\section{Determining the Optimal Policies for Boosting FDI Flows in Canada post-COVID-19 using a Preliminary Analysis from the SARS Pandemic}

\section{Abhishek Chatterjee, Alena Ho, Silvanus Kolade, and Siddharthan Lakshmanan}

University of Toronto

As Canada transitions towards a post-COVID-19 society, the pandemic has left the Canadian economy disrupted. To stimulate the process of economic recovery, we look towards proposing potential foreign investor policies to increase sector specific FDI, targeting the Manufacturing, Professional,
Scientific, and Technical Services, and Transportation and Warehousing sectors. In particular, we hypothesize that FDI and real GDP growth have a direct positive correlation during times of economic crisis. We explored this correlation by graphing data analyzing real GDP growth, net FDI flows, and current/capital account balances during the SARS epidemic. By reflecting on Canadian and Chinese data provided during the SARS pandemic, we can infer the trends for each sector and provide optimal policies to maximize foreign investment. These novel solutions were inspired in part by current policies implemented in other parts of the world, which we put into a Canadian context. They focused on encouraging early graduation and increased incoming students into post-secondary institutions, increasing career qualifications in unemployed adults, regulating the influx of traffic when quarantine policies are reduced, and providing extended work permits to substitute for the decrease in the current labour force. Furthermore, our findings serve as a general frame-work for international government policy, which can be built upon for future epidemics.

Mentor: Marichelle Leclair

Keywords: COVID-19 crisis, SARS pandemic, FDI, Sectors, Policies

\section{An Investigation into the Relationship Between Malarious Countries and COVID-19 Cases}

\author{
Anne Jing, Vimal Raj, and Charles Liu \\ University of Toronto
}

Studies have shown that a negative correlation may exist between the number of COVID-19 cases and the incidence of malaria. This relationship is important, as it can provide valuable insight into potential treatments of the virus. To further investigate the correlation, 66 other factors (such as country demographic, GDP per capita, Health Index etc.) were examined. Open source datasets were collected online from institutions such as the John Hopkins Coronavirus Resource Center and analyzed using various Python libraries. By calculating various correlation coefficients, it was determined that the number of tests conducted, air traffic and other several other factors correlated with COVID-19 cases, and may be responsible for the lower number of COVID-19 cases 
instead. To further strengthen the results, a machine learning model was constructed to evaluate the most relevant factors contributing to the transmission of COVID-19. Here, malaria was not deemed as an important feature. Instead, factors such as climate and country wealth were most important. A mixture of statistics and machine learning helped show that various factors unrelated to malaria may be responsible for the lower occurrence of COVID-19 in malarious countries, and while this is not enough to prove causation, it makes it likely that the correlation between COVID-19 and the Plasmodium parasite is spurious.

Mentor: Sanjai Arumugam Jaganmoham

Keywords: COVID-19, malaria, machine learning, cases

\section{Analysis of Coverage of BCG Vaccine and its Impact on COVID-19}

\section{Agnes Li, Crystal Qin, Akim Ruslanov, and Claire Wu}

\section{University of British Columbia}

Google search interest towards Bacillus Calmette-Guérin (BCG) Vaccine peaked be- tween April 5th and 11th, 2020. At the same time, the number of confirmed cases of COVID-19 topped 400,000 in the United States alone [1]. Although there is no evidence that BCG vaccine can protect from COVID-19 infection, there is still a keen interest in how the vaccine can impact the spread and mortality rate of COVID-19 in a given community. This study aims to find if there is a statistical significance in relation between BCG vaccine and COVID-19 by comparing average infection rate and average mortality rate of countries divided into three groups: 1) BCG coverage is universally present, 2) BCG coverage was universally present, but not at the moment, 3) BCG vaccine was never widely used [2]. The study has found that in countries where universal BCG coverage is present, the infection rate is significantly lower compared to the countries where BCG coverage was previously universal and where BCG coverage was never present.

Mentor: Aroop Ghosh

Keywords: Covid-19, BCG vaccine, BCG coverage
The Interdependency of Policy, People, and COVID-19: An Analysis using Time-Lagged Cross Correlation

Aubrey Maltz, Daid Ahmad Khan, Geoffrey Siow, and Haritosh Patel

University of Waterloo, Harvard University

COVID-19 has had a profound impact on the way governments function and on population behaviour. However, these changes are not without reason; they are motivated by a need to flatten the curve and mitigate loss of life. In this work, it is hypothesized that three timevarying datasets: University of Oxford's OxcGRT stringency index, Google's Community Mobility Reports, and daily new cases of COVID-19 per capita, exhibit interdependency. The connection between these three time series datasets is established using Pearson correlation on select countries. These countries are categorized by their promptness to implement stringent policies. Expanding upon this, potential causal relationships between stringency, mobility, and new cases are established using time-lagged cross correlation. This two-dimensional analysis is further expanded by using windowed time-lagged cross correlation to investigate how causality and correlation between these variables evolves with the progression of COVID-19. Time-lagged correlation analysis indicated that while mobility and stringency appear to be consistently negatively correlated, as can be expected. However, correlations were more complex for both casesstringency and cases-mobility relations. The analyses in this work indicated varying time dependencies between mobility and new cases. Government stringency correlation with new cases was found to invert from positive to negative correlation as the pandemic progressed suggesting harsh government policies can curb the spread of COVID-19, albeit after a period of delay. This time-lagged cross correlation analysis could be further developed with the integration of other factors such as testing numbers and contact tracing policy.

Mentor: Amin Mawani

Keywords: coronavirus, income, population, race, ethnicity, vulnerability 


\section{Analysis of Socioeconomic Determinants of Increased COVID-19 Vulnerability}

\author{
Alexandra Mircescu, David Cao, Pramith Senaratne, \\ and Sajeev Kohli
}

McGill University, Harvard College, University of Michigan

COVID-19 has undeniably impacted many populations around the world, but some regions and populations are hit harder than others. This study seeks to examine some of the most commonly cited socioeconomic indicators for COVID-19 vulnerability, and statistically evaluate how much they influence COVID-19 incidence. The study looks into household income, population density, race and ethnicity as potential indicators of COVID-19 vulnerability. Relevant data was extracted from multiple sources, including county level COVID databases, Zip Atlas, US Census, and the COVID-19 Tracking Project. To evaluate household income and population density, linear regression plots at a zip code level for counties in New York City and the Bay Area were created to visualize correlation. For race and ethnicity, ANOVA tests were used to confirm statistically significant differences between COVID-19 incidences and fatality rates between differing racial and ethnic populations. PCA Analysis through singular value decomposition and a combination of K-Means clustering, hierarchical clustering and Gaussian Mixture Modelling clustered states with similar racial and ethnic distributions in COVID-19 incidence and death rates together. Linear regression models were made for individual clusters to assess the relationship between percentage makeup of state population and percentage makeup of COVID-19 incidence. Household income regression plots confirmed a common downward correlation where increased household income led to decreased COVID-19 incidence and death rate. Population density seems to have no clear relation with COVID-19 incidence. Racial and ethnic distributions clusters seem to be linked geographically. On average, African American individuals seem to have a higher rate of incidence and fatality than caucasian and Asian individuals for the same makeup of state population. Similar trends were seen at a smaller geographical scale looking at Florida and its counties. In the end, the study provides a new way of understanding commonly cited socioeconomic indicators for COVID-19 vulnerability, and allows future researchers to more efficiently identify at risk groups and populations before, during and after similar pandemic cases.

Mentor: Sydney George

Keywords: COVID-19, Policy, Population Mobility, Cross Correlation, Stringency

\section{Correlation Between SES and Obesity in Canadian Adults}

\section{Ashmita Mazumder and Farhat Ullah}

\section{University of Toronto}

We aim to examine the complex relationship between socioeconomic status and body mass index of adults in Canada. High BMI is a major risk factor for various diseases like diabetes, asthma, high blood pressure etc. Understanding the relationship between socioeconomic status and body-mass index can help target vulnerable populations and implement public health decisions with much more confidence. Data from the Canadian Community Health Measures Survey 2019 was used for analysis in this paper. This dataset was accessed through the open data portal of Statistics Canada. We hypothesized that both education and income would have a significant negative correlation with BMI. The level of education and total household income of respondents were used as indicators of SES. Results demonstrated a complex relationship between the variables. We found significant correlations for both education and income although for education we found a significant positive correlation. Our results suggest that there may be mediating factors like gender, marital status and cultural identity among few that contribute to these relationships.

Mentor: HuyThong Bui

\section{Investigating the Effectiveness of Strict Government Policies in Decreasing the Incidence of COVID-19}

Andrew Pringle, Christine Orcullo, Adam Tinaburri, and Seokhyoun Hong

Simon Fraser University 
The COVID-19 pandemic has revealed the true state of a nation's emergency preparedness and flaws within individual countries' government systems. Although there are general guidelines set by the World Health Organization (WHO) contingency planning for such an event, the efficacy and transparency of different levels of government intervention to mitigate the spread of the disease have varied the incidence rate and general timeline of the disease worldwide. The purpose of our analysis is to investigate the changes in the incident rate of COVID-19 and its projected timeline of cases concerning the degree of government intervention imposed. Based on a Stringency Index, reflecting the strictness of government policy put in place, and filtering the countries based on GDP per Capita, we separated countries into groups to compare the effectiveness of government policy. After curve fitting two-term gaussian curves to each country, we were able to find and compare the elapsed time since enforcement until the projected mitigation of the virus. We tested the difference in means of elapsed time between groups differing in government policy. This was done using two-sample t-distribution hypothesis testing to a 0.05 significance level. The results showed a lack of significant evidence to suggest the correlation between strict policy imposition and the decrease in incidence rate. The effectiveness of government policy in controlling the spread of a virus is impeded by numerous confounding variables such as population density, reliability of data, and social determinants of health. Therefore, future studies may examine such variables to test the validity of the use of strict policies in countries.

\section{Mentor: Mohammad Mamun}

Keywords: COVID-19, policy, government, Stringency Index, gaussian curve

\section{An Analysis of US Health Insurance and H1N1 Vaccination Coverage: Implications for a COVID-19 Vaccine}

\author{
Anaïs Rojas Vélez, James Li, Ziniu Chen, and Yen \\ Ching Tan
}

University of British Columbia, McGill University

The upcoming COVID-19 vaccination campaign will be vital.
Here, the H1N1 influenza pandemic is used as an indicator of steps that can be taken to maximize deployment of such a vaccine. We used data from the 2010 National Health Interview Survey (NHIS) in our analysis $(n=16027)$. Having health insurance was significantly correlated with H1N1 vaccination. Having no coverage had an odds ratio of 0.384 (95\% Cl 0.338 - 0.436) for H1N1 vaccination compared to insured individuals. Uninsured individuals were up to 2.6 times less likely to have been vaccinated. A large portion of US health insurance is reliant on employer-sponsored coverage, both in the 2010 data and in more recent trends. The massive employment loss caused by the COVID-19 pandemic and the corresponding loss of coverage suggests the US should take steps towards universal funding of the COVID-19 vaccine once it becomes available.

Mentor: Danielle Roy

Keywords: health insurance, H1N1, COVID-19, vaccination coverage, unemployment

\section{The Impacts of COVID-19 on the Black Community in the United States of America}

\section{Aditya Singh, Flora Zhang, and Nishita Dahisaria}

\section{University of British Columbia}

The Coronavirus is a pandemic that has quickly taken over the entire planet. It's impact has been most pronounced in USA, with the states having both the highest number of confirmed cases and deaths at the time of writing[1]. However, when examining data regarding the effect of the pandemic on different races, multiple findings reported a higher mortality rate amongst black people than any other race. This study aims to discern the reasons behind this discrepancy. This problem was investigated using R and MS Excel to analyse open data-sets and investigate possible relationships. Multiple regressions were performed to figure the factors that significantly affected the black population death percentage. Our findings suggest Black population\%, Population density, Median House Value, Life Expectancy, and Diabetes Prevalence to be those factors. On the other hand, Poverty was a surprising omission. As only data from states with above-average black populations was used, it would be interesting to see this study replicated using data from all the 
states of the US. It is unknown if the pattern acknowledged by this study is prevalent in other countries as well. Therefore, a similar study conducted on other countries with significant black minorities may also be a worthy endeavour.

Mentor: Owen Whitley

Keywords: COVID-19, Coronavirus and Race, Socioeconomic and Health Factors, Black People in the USA

\section{An Ensemble Matrix-Factorization-Based Recommender System for Predicting the Effectiveness of Antidepressant Drugs}

\section{Alun Stokes and Mehrunnisa Shiraz \\ McMaster University}

With increases in the prescription of psycho-pharmaceutical drugs, the necessity of effective methods for determining which drugs to prescribe is immense. Further, the highly variable and potentially severe reactions that patients have to these classes of drugs indicate the need for a more personalized approach to the prescription and management of such drugs. Response to early treatment is known to highly affect patient response to continued treatments, with positive early experiences improving outcomes. As such, being able to efficiently determine the correct drug choice is paramount to improving patient well-being. To this end, we propose the use of an ensemble matrix-decomposition-based collaborative filtering model to predict how a patient will react to a drug they have not tried, as determined by their reactions to previously tried drugs. This is done by using information from how other patients have reviewed the various drugs they have used. In this paper, we examine this concept with respect to antidepressants, and their use in the treatment of depression, anxiety, insomnia, and obsessive-compulsive disorder. We report $0.78,0.74$, and 0.72 fraction of concordant pairs in the prediction of best-choice drugs for depression, anxiety, and insomnia respectively. Most impressively, our method of collaboratively filtering allows accurate predictions from very minimal information, with patients only having a single drug rating being given reasonably accurate predictions. Finally, the model becomes more accurate as a patient has additional drug ratings, allowing an appropriate drug to be identified very quickly.

\section{Mentor: Yasaman Amannejad}

Keywords: collaborative filtering, matrix decomposition, ensemble learning, personalized healthcare, antidepressant drugs

\section{Exercise and Sedentary Behaviours of Average North Americans during COVID-19}

\section{Abhirup Shah, Sebastian Woo, and Arjunn Sivakumar}

Wilfrid Laurier University

This study examines the effects of COVID-19 on the physical sedentary behavior of the general public in North America. Prior to launching this study we had not found any peer reviewed articles or datasets pertaining to this topic and believed this would be a suitable study to pursue that made use of both open data sets and alternative data sources. Data was sourced from Google Trends, Yahoo Finance, Government of Canada, and Steam open data. Google Trends data was scraped for keyword frequency levels from North America across a twelve month period to assess the sentiment towards fitness and activity. Earnings reports on publicly traded health fitness companies were derived from Yahoo Finance to evaluate sales performance pertaining to equipment used for personal home fitness. Open datasets from the Government of Canada published by Statistics Canada were used to evaluate general sedentary behavior levels prior to COVID-19. Open data from video game software Steam was used to track sedentary behavior throughout social isolation. What we found was that throughout a one year period, attitudes toward fitness and exercise were consistent and showed a flat curve up until the second week of march which yielded a greater sentiment towards physical activity. We also found that throughout a six year period, sedentary behavior was on a year over year decline but suddenly jumped throughout the month of March but quickly subsided. Evaluating the data and findings, we conclude that fitness throughout the North American population had improved throughout the social distancing of COVID-19 but physical sedentary behavior had dramatically increased but is gradually subsiding.

Mentor: Mohammed Mamun

Keywords: Google Trends, StatsCan, COVID-19 


\section{Correlation between COVID-19 cases in African Countries and Flights}

\section{Akrash Sharma, Tanyaradzwa Gozhora, and Xiang Zhang}

\section{University of Alberta}

The growth of the global COVID-19 pandemic has been relatively slow in many African countries, potentially due to lesser international travel to major cities compared to other continents. The goal of this paper is to determine the extent and type of the relation between international flights and cases of COVID-19 observed currently in Africa. The observations could show where the focus of countries should be between initiating lock downs and imposing border restrictions. This analysis was done through clustering and linear regression modelling for the clusters. Significant correlation was observed during the analysis.

Mentor: Michael Dorrington

Keywords: COVID-19, Coronavirus, Africa, Flights, Policy

\section{A Data Analysis of the Changing Social Communications during the COVID-19 Pandemic and Subsequent Social Consequences}

\section{Anna Xia, Helena Teng, and Rijuta Gohil \\ McMaster University}

There has been a dramatic shift towards online communication platforms since the outbreak of COVID-19 and the subsequent introduction of physical distancing in Canada. The ability to access these online networking resources is crucial to staying connected during these unprecedented times; however, there may be certain groups that face barriers to online communication. This article identifies some of these extraneous groups, while describing the possible consequences they face as a result of limited channels communication, specifically focusing on the correlation between social connection and mental health. Datasets were obtained from Statistics Canada and refined using R. Through the analysis of the datasets, we conclude that the elderly have the least knowledge on how to use the internet. It was also found that those with lower household incomes have the least access to the internet in Canada. These findings suggest that a lack of knowledge and insufficient access are barriers to effective online communication and may result in increased social isolation. This is especially critical, as the data shows online resources to be in higher demand during the COVID-19 pandemic. Furthermore, social isolation as defined by the "inadequate quality and quantity of social relations with other people at the individual, group, community, and larger social environment levels where human interaction takes place" has been linked to depression and social anxiety disorder [1],[2],[3]. This article highlights the importance of communication between individuals and how COVID-19 has affected it. In the future, this research can be repeated with more data regarding communication and qualitative surveys from populations during COVID-19.

Mentor: Daiva Nielsen

Keywords: COVID-19, mental health, social isolation, communication, social media

\section{Policy, Demobilization, and Infection: A Multilateral Approach to Social Distancing}

\section{Alexander Y. Liebeskind, Amy MiHyun Jang, Thomas Tran, and Nikhil Mehta \\ Columbia University}

Over the course of the 2020 COVID-19 pandemic, social distancing has proven to be one of the most effective and prevalent methods of mitigating viral spread. Social distancing policies implemented in different countries, however, have widely diverged in strictness and area of focus and have therefore catalyzed varying responses in population mobility. The effects of specialized changes in social distancing policy are still largely unknown or unvalidated. This study aims to address this gap in comprehension by incorporating independently sourced data on COVID-19 infection, government action, and population mobility into a consolidated analysis of social distancing. Results indicated that social distancing policies targeting containment/ health were correlated with rapid, significant decreases in population mobility, while policies directed at economic support failed to produce a quick or statistically significant change in population mobility. Furthermore, stricter and 
more robust social distancing policies were discovered to be more effective than less austere policies for some but not all areas of focus. These findings provide vital insight into the nuanced repercussions of individual changes in social distancing policy. This information is advantageous in the alteration and creation of future social distancing policies.

\section{Mentor: Priyanka Gogna}

Keywords: social distancing, data visualization, health policy, epidemiology, biostatistics

\section{An Epidemiological Approach to Evaluating the Relationship between a County's population Density and its Ability to Practice Physical Distancing}

\author{
Bowen Ma, Naitik Acharya, Taha Elghamudi, \\ Talha Syed \\ University of Guelph
}

The world is currently in the midst of a pandemic. The spread of severe acute respiratory syndrome coronavirus 2 (SARS-CoV-2) has resulted in record high numbers of infections across many countries, particularly North America. In an effort to lessen the detrimental impacts of the novel coronavirus disease (COVID-19) and the spread of SARSCoV-2, public health officials have introduced interventions such as physical distancing. Physical distancing is a method of non-pharmaceutical interventions with the intention of dampening the spread of contagious diseases by maintaining a certain physical distance from others and reducing the number of close contacts. In fact, physical distancing has been successfully implemented in the past to curtail the spread of several pandemics across multiple decades. However, unlike past pandemics, the human population within different counties and cities has grown exponentially which can make it difficult for citizens to practice physical distancing. Therefore, the aim of our study is to investigate the ability of counties with different population densities within the United States to practice physical distancing through the analysis of physical distancing grades and analyzed using tools such as a multivariate regression in R. In our analysis, we have shown that counties with smaller population densities are better at mitigating close encounters while worse at decreasing the frequency of non-essential visits. Overall, counties of different population densities, however, do seem to differ in their ability to practice physical distancing, i.e. their physical distancing grade, which opens new avenues for density specific regulations while supporting a national preventative approach.

Mentor: Omar Shafiq

Keywords: COVID-19, Social Distancing, Public Health Policy

\section{The Impact of COVID-19-related Unemployment on Suicide Rate in Canada}

\author{
Catherine Chen, Denny Liu, and Nan Ji Suo \\ University of Toronto, Western University
}

Suicide is the 9th leading cause of death in Canada, resulting in approximately $\mathbf{4 0 0 0}$ deaths per year. Many factors contribute to changes in suicide rates, including socioeconomic and demographic factors in addition to individual health and personality characteristics. With the current COVID-19 pandemic producing record-high unemployment in Canada, as well as globally, this paper aims to explore the association between suicide and unemployment, and estimate the additional impact of COVID-19 on suicide rates. Using historic suicide and unemployment data from 1979-2019 stratified by age and sex, a positive relationship between suicide rate and unemployment rate was determined. The linear regression model was then used to predict the suicide rate in 2020 using various unemployment rate scenarios. The largest impact is observed among males aged 35-54, with a moderate COVID-19 impact expected to contribute to an additional 75 suicides, increasing to 210 additional suicides under the severe COVID-19 scenario. For males aged 25-34, the result is similar, with 70 to 180 additional suicides predicted. The impact is slightly lower among females, with an impact of 60 to 190 additional suicides among older women aged 3554 compared to 55 to 155 additional suicides among females aged 25-34. The findings of this paper highlight the mental health implications, in addition to the economic burden, resulting from the COVID-19 measures, and suggest a strong need for the Canadian government to pre-emptively invest in mental health support programs and other suicide prevention strategies. 
Mentor: Amin Mawani

Keywords: COVID-19, coronavirus, mental health, suicide rate, unemployment rate

\section{Dependence and Depression Levels in Residential Care Residents Throughout Canada}

\section{Celeste Groux}

McGill University

It is no secret that the population of Canada is aging. Statistics Canada estimates that in 2019 there are more than 6.5 million people above the age of 65 , accounting for $17.5 \%$ of total population estimates. As this group of the population grows, so too does the need for residential care in places such as nursing homes and long-term care facilities. This study looked at dependence and depression levels of individuals in residential care across Canada according to the ADL Performance Hierarchy Score, as well the Depression Rating Scores and depression diagnoses. The correlation between depression diagnoses and dependence levels was then analysed. Data which provided information on individuals receiving publicly funded/subsidized residential care for 2016-2017, 2017-2018, and 2018-2019 was obtained from the Canadian Institute for Health Information. This data was combined to obtain mean values for studied features across the three years provided. Alberta, Ontario, Saskatchewan, British Columbia, Manitoba, Newfoundland and Labrador, and Yukon were all included in over 1000 facilities. It was found that Ontario and Alberta had highest proportion of individuals with high ADL scores and individuals diagnosed with depression. For the entire population assessed, $46.9 \%$ of residents were found to have a 5 or 6 ADL Score and thus very high dependency levels. Also, Manitoba had the highest proportion of no depressive symptoms, while Saskatchewan and British Columbia had the lowest percentages of depression diagnoses. Most notably, a moderate negative correlation was found between low levels of dependence and depression diagnoses, while a high correlation was found between ADL Score 4 and depression diagnoses in those receiving residential care.

Mentor: Yasaman Amannejad
Keywords: Aging, Dependence, Depression, Residential Care

\section{Estimating the Effectiveness of Improvements in Test Process: A Modelling Study}

\section{Charles He and Jingyuan Lu}

Western University, University of British Columbia

The test process of coronavirus disease 2019 (COVID-19) affects the transmission. The process is mainly determined by the following factors: the regional maximum test capacity, the waiting time in queue and the average number of days to get test results under current technological condition. Based on the real situation of Canada, We quantify these factors as a random variable, then introduce it into the work of Hellewell [1], which focus on the effect of isolation of cases and contacts, to analysis the effectiveness of improvements in test period in Canada. After the enhancement the transmission model (Hellewell, 2020), We quantify the potential effectiveness of improvements, including extension of test capacity, evolution of test techniques and investment on human resources like training and call of volunteers. We consider scenarios different in the number of initial cases, the time period of symptom onset to test, the contacts tracing. We ignore the time period of getting test result to isolation, and assume isolation will reduce any further infection. For each scenario, we run the test and judge the controlling result by total cases. As the Government of Canada introduced new test techniques and extend regional test capacity, our modelling study analysis shows that the simulated outbreaks would be better controlled. This result matches the empirical result in Canada. Other policies, including isolation, legislation on prohibiting organized public events and social gatherings more than a number of people, and case tracing, are also necessary to the controlling of pandemic.

Mentor: Christy Tomkins-Lane

Keywords: COVID-19, Test period, Test techniques

The Effect of Health Crises on Child and Maternal Mortality: A Comparative Analysis on the Ebola Outbreaks in Guinea, Liberia, and Sierra Leone 


\section{Caitlyn Tran, Ethan Seto, Rishabh Chakrabarty, and Priscilla Maryanski}

University of California, University of Pennsylvania, Manipal Academy of Higher Education, New Jersey Institute of Technology

While past studies only use data about the health crisis they are dealing with to guide intervention, this can lead to late action or too many assumptions to be accurate. Hence, the present study creates a statistical model to guide future preparation for facing health crises based on the effect of different intervention coverages during the 2014-2016 Ebola outbreak in the West African countries Guinea, Liberia, and Sierra Leone. This was accomplished with the Lives Saved Tool (LiST), by measuring the number of more lives saved and the decrease in child and maternal mortality if the higher intervention coverages that occurred in Liberia and Sierra Leone were implemented in Guinea. The results were used to establish thresholds with readiness levels. Each readiness level represents either Workforce \& Services or Supplies \& Equipment, the two aspects of a nation's healthcare system as defined by this study. Compared to the mortality rates and lives saved in Guinea with no interventions, Threshold 1 (expected results if Guinea had implemented Liberia intervention coverages), Threshold 2 (expected results if Guinea had implemented Sierra Leone intervention coverages), and Threshold 3 (expected results if Guinea had implemented both Liberia and Sierra Leone intervention coverages) increasingly reduced mortality rates and saved more lives. These thresholds, composed of calculated numerical readiness levels, enable policymakers to set concrete goals for preparing for future health crises, which are ever increasing as a result of globalization and technological innovation. The present study establishes a foundation for future researchers to create better informed approaches for health crises.

\section{Mentor: Aroop Ghosh}

Keywords: health crises, child mortality, maternal mortality, Ebola, West Africa

The Impacts of International, Domestic, and Local Mobility on COVID-19 Deaths
Cathy Yan, Simran Pal Waraich, and Liza Babaoglu

University of British Columbia, Simon Fraser University, University of Toronto

As coronavirus disease 2019 (COVID-19) spread across the world, countries restricted travel and mobility at the international, domestic, and local levels. However, these new policies have devastated the global tourism industry and faced resistance from people of all backgrounds. Due to the unprecedented nature of this global crisis, investigating the efficacy of these restrictions is important for future decision making by policymakers and individuals. Using datasets from the United Nations World Tourism Organization (UNWTO), Google, and John Hopkins, the relationships between mobility and the spread of COVID-19 was evaluated using linear regression and K-means clustering. It was found that having fewer people enter the country correlated with a lower number of COVID-19 deaths, and visitations to local indoor public places were associated with a higher number. However, no conclusions were drawn between death rates and tourism-related trips taken within a country, or with outings to parks. These findings, in general, support restrictions on international travel and stay-at-home orders, but will hopefully provide insights and opportunities for more nuanced guidelines that support economic and social needs without compromising public health.

\section{Mentor: Sean La}

Keywords: Coronavirus, Travel, Tourism, Big Data, Machine Learning

\section{Maternal Literacy and its Effect on the Nutritional Status of Children}

\section{Danya Alomar and Dalya Alomar}

\section{University of Ottawa}

The health and nutrition of children at a young age is critical for their proper growth and establishment of their physical and mental abilities. It is therefore of considerable scientific interest to determine a child's optimal dietary requirements and to combat nutrient deficiency along with its consequences on growth and development. Understanding the causes of malnutrition by conducting research analysis is a key step to finding effective solutions to a healthier generation. The 
objective of this study is to analyse the underlying cause of the three forms of child undernutrition- stunting, wasting and under-weight. In the context of addressing malnutrition, alongside other key development measures, women's education is often highlighted as the largest determinant of positive outcomes [1]. As such, the literacy levels of adult females in various countries were collected from multiple data portals including the World Bank, Our World in Data and UNICEF to develop the following graphs: a scatter plot to demonstrate the effect of literacy on malnutrition of children in select countries, a line chart with multiple linear regressions to display the trends of literacy and children malnutrition in the long term, and a geographic map to visualize areas most affected by stunting. These findings lay evidence that countries with higher female literacy rates tend to have lower stunted, wasted and underweight children. Subsequent analysis reveals a positive relationship between female adult literacy and the nourishment of young childrena higher level of literacy on the mothers' part can greatly impact the proper nourishment of children under the age of five. The pathways highlighted by this study include the need of improving access to nutritional education and counselling programs for mothers in order to make safe health and diet choices for their young children. While maternal literacy is not necessarily the only factor for undernutrition, it does have strong correlations with the health and growth of young children. As an area of future research, this topic could further explore the correlation between children malnutrition and factors other than parental education in order to establish which is the greatest contributing factor.

\section{Mentor: Aliyah Dosani}

Keywords: child malnutrition, stunting, maternal literacy, educational intervention

\section{Early Detection of Cognitive Decline}

\section{David Cho, Diana Khater, Fiona La, and Jordan Lam}

University of Waterloo

Cognitive decline is a prevalent problem in today's health industry commonly associated with an aging population. The biggest age group in America is 55+, reported to be 29 percent of the total population in 2018. This means that a large portion of the American population is more prone to experience cognitive decline over time. Cognitive decline is the decreased performance of cognitive ability in individuals which often leads to greater symptoms and neurological diseases such as dementia and Alzheimer's which have no present intervention or cure. This impacts not only the memory and thought of an individual, but can also hinder their day to day functions. It is annually estimated that 1 and 9 Americans experience subjective cognitive decline. The best way to combat these problems is through early detection and preventative measures, through cognitive stimulation therapy and pharmaceutical measures. Variables such as age, sex, level of education, physical activity, and obesity are factors that have been seen to contribute to the onset of Alzheimer's. The scope of this project is to create a probability model regarding the factors to help concerned individuals compare and reflect their personal factors and determine their susceptibility to cognitive decline which encompasses dementia and Alzheimer's. This probability model would aid in early detection, the best method for prevention. The probability model was constructed using the Baye's probability theorem, which bases the probability on an event based on prior conditions.

Mentor: Wanhua Su

Keywords: Dementia, Cognitive Decline, Baye Theorem, Early Detection, Preventative Healthcare

\section{Systematic Comparison Between Varying Safety Measures in the United States of America and South Korea During the COVID-19 Pandemic}

\section{David Cai, Kevin Nishimura, and Daniel Tan \\ Western University, The University of British Columbia}

The sheer impact of the COVID-19 pandemic on the global community is unequivocally immeasurable. One of the most divisive issues of this pandemic is the uncertainty surrounding the efficacy of the safety measures that have been imposed by countries around the globe. Using the datasets collected from organizations including the Organisation for Economic Cooperation and Development (OECD) and the Assistant Secretary for Planning and Evaluation (ASPE), this paper aims to evaluate and compare the varying safety measures of the 
U.S. and South Korea. More specifically, the central target of such analysis is to propound the successful safety measures imposed by the South Korean government to the relatively ineffectual U.S. government. This analysis is performed using data comparison and statistical inference using Python and is further backed by predictive models pertaining to the COVID-19 pandemic. By narrowing the target demographic to those who fall below the poverty line of their corresponding country, the analysis yielded two notable safety measures which are highly encouraged to be undertaken by the U.S. government: an increase in government funds and food programs.

Mentor: Hannen Abbas

\section{The Role of State-wide Stay-at-home Policies on Confirmed COVID-19 Cases in the United States: A Deterministic SIR Model}

\section{David Chen, Seungmin Lee, Summers Wu, and} Jason Sang

University of Western Ontario, University of Toronto, University of Waterloo

In January 2020, the first confirmed case of the novel severe acute respiratory syndrome coronavirus 2 emerged in the United States of America. By March 2020, the USA had declared a national emergency and implemented stay-at-home policies subject to the individual initiative of health authorities of each state. However, ambiguity in the literature exists about the extent to which temporal variation of stay-at-home implementation contributes to an effective stay-at-home order. To examine the role of the implementation of stay-at-home policy at the county level on outbreak progression, we compiled the case count data and dates of policy commencement for 1720 counties from the US Counties: Socio-Health Data database. Measures of central tendency and rate of change identified correlation between the change of confirmed case counts compared to time, quantified by comparing four successive time points of 5 days to the initial date of each county's stay-at-home implementation. We then used a deterministic county-level SIR epidemiological model to predict post stay-at-home case counts based on pre-stay-at-home parameters and compared the model to actual post-stay-at-home case counts to identify the degree of error Mean Squared Error (MSE). Our analyses demonstrated the high error between time since stay-at-home implementation and change in actual case counts compared to predicted case counts, which suggests an interaction between policy and COVID-19 transmission. Our findings shine light on the confounding variables of stayat-home policy at the county level and the promising outlook of stay-at-home policy in the USA.

Mentor: Vishal Satyanvinayak Purohit

Keywords: stay-at-home, SIR model, deterministic

\section{Effectiveness of Decreasing Public Mobility as a Primary Method of Flattening the COVID-19 Curve}

\section{Dayoung Kim and William Shen}

University of Calgary, University of British Columbia

Through a county level examination, this paper seeks to evaluate the effectiveness of U.S partial lockdown measures in controlling the spread of COVID-19. Datasets on the number of daily new cases and the percentage changes from baseline mobility up to May 16th were collected from USAfacts.org and Google Mobility respectively. From the first COVID-19 incidence in a county, a cubic regression was used to create a model to describe the number of new cases occurring each day. The cubic model was used to interpolate the day of the maximum number of cases or peak, with the exception of counties where the peak has not been reached and thus had to be extrapolated. The interpolated or extrapolated dates for each county was then complied together to perform a linear regression between the number of days until the peak and average changes to baseline mobility until the peak occurred. Residuals and $\mathrm{R}$ squared was used to judge the quality of the model. These methods resulted in observing a weak correlation between the average change in baseline mobility and the number of days taken for new COVID-19 cases to peak. As the average mobility increased from baseline, the days for new case to plateau decreased. While affirming the influence of mobility decrease in decreasing the rapidity of COVID-19 spread, the weakness of correlation suggests the need for a multifaceted public health policy. Furthermore, the various 
ranges in average percentage changes to baseline mobility reveals that federal lockdown measures are implemented inconsistently across counties. In order to determine public policy that takes into account he specific circumstances of the counties to produce the most effective control, future studies must be done on the influence of factors such as population density, social attitudes, mask-wearing behaviours to gain a better grasp on why changes in mobility are effective to such varying extends among counties.

Mentor: HuyThong Bui

Keywords: Public Mobility, COVID-19, Flattening the Curve, Partial Lockdown

\section{Flatten the Curve, Save Lives: Exploring the Effectiveness of Intervention Methods on the Spread of COVID-19}

\author{
Dylan Miller, Nagam Saleh, and Jordan Slessor \\ MacEwan University
}

COVID-19 is a new threat that governments are facing on a global scale. One of the main focuses surrounding the COVID-19 pandemic is in reducing the amount of cases a region has, which has been termed as "Flattening the curve". There have been many different approaches to flattening the curve with varying effect. In this paper we compare intervention methods proposed and practiced by several governments to determine the most effective way one can "Flatten the curve". Our hope is that in determining the most effective intervention, the global impact of the COVID-19 pandemic will be greatly reduced. Using datasets on confirmed cases around the world, as well as research done on intervention methods of the corresponding countries, we were able to identify which intervention scenario was most effective. We found that mean reproduction rates were significantly different $(F(7,91)=5.425, p<0.001)$ amongst 8 countries/ regions that used different intervention strategies. Despite using similar intention strategies,

Mentor: Randa Higazy

Keywords: Coronavirus, COVID-19, Government Intervention, Reproduction Rate, ANOVA

\section{Correlation between Cases of COVID-19, Related Media, and Public Risk Perception, in relation to the H1N1pdm09 Pandemic in the UK.}

\author{
Sufal Deb, Carmina Isidoro, Kian Yousefi Kousha, \\ and, Marvin Yan
}

McMaster University

In the midst of the COVID-19 pandemic, the media has become the general public's primary and sometimes only source of information during the unexpected disconnection caused by quarantine and social distancing. The influence of media can be duplicitous - particularly during our current climate, with respect to how people perceive the pandemic. This paper aims to determine if the media coverage of COVID-19 has influenced the risk perception of the United Kingdom populace. Using public response to the previous $2009 \mathrm{H} 1 \mathrm{~N} 1$ pandemic as a comparator to the COVID-19 situation, we used a selection of datasets, extracted from several sources including the EU Open Data Portal, and the Johns Hopkins Coronavirus Resource Center, both of which were analyzed through statistical methods such as linear regression and chi-square test. The analysis of the H1N1 pandemic indicated that the number of associated media stories published peaked earlier than the number of confirmed infection cases. Moreover, an analysis between media stories and $\mathrm{H} 1 \mathrm{~N} 1$ cases followed a negatively sloped trendline. This asynchronicity between the two curves is a likely explanation for the significantly low risk perception of H1N1 in surveys. This reduced risk perception is considered to have been a key element in poor vaccine uptake in the UK. The conclusions from the H1N1 analysis were used to evaluate and make predictions about the COVID-19 pandemic - particularly as COVID-19 related media coverage has shown a significant decrease since March 25, 2020. Recent surveys have also shown a significantly high risk perception for COVID-19, introducing a great window for intervention. In fact, an increased and consistent level of media coverage on the pandemic may keep public risk perceptions high - potentially culminating in more receptive attitudes towards vaccine uptake when available. Despite these results, the novelty of the situation leaves some uncertainty to be explored in the future. 
Mentor: Jenny Bicong Ge

Keywords: COVID19, H1N1, media, risk perception, pandemic

\section{Can Respiratory Disease Mortality Rate Predict the Difference between Canada and England's COVID-19 Mortality Rate: a Comparison Study}

\section{Dafer Tania, Du Iris, Mrakade Gio, and Song Kevin}

McGill University

Since the emergence of severe-acute respiratory syndrome-related coronavirus (SARS-CoV-2) in Wuhan, China in December of 2019, this deadly virus has rapidly spread across nations, affecting 188 countries and resulting in over 370,000 deaths worldwide. As a respiratory disease, COVID-19 is characterized by symptoms of fever, cough and difficulty breathing, and can lead to pneumonia. In this paper, we hypothesize that a higher rate of respiratory disease mortality may predict higher rates of COVID-19 mortality. We analyzed mortality rates of England and Canada in regard to both respiratory conditions and COVID-19. Although both countries are similar in their accessibility to their respective health care systems and population age cohorts, their governments took different approaches in the early stages of the pandemic, resulting in drastic differences in COVID-19 cases to date. Upon analysis, England was observed to have higher mortality rates for both respiratory conditions and COVID-19, indicating a potential relationship between the two mortality rates. Further analysis of additional countries would be required to establish a statistical correlation between the two conditions. However, if proven to be related, data trends on respiratory disease mortality rate could potentially indicate which countries would be the most at-risk for COVID-19 mortality. This information can be used to prioritize access to a safe and effective vaccine against SARS-CoV-2, when it becomes available, for certain countries and populations within.

Mentor: Robert A

Keywords: COVID-19, Respiratory Disease, Mortality Rate, Canada, England

\section{Exploring the Correlation Between Depression and Obesity Across the World}

\section{Daniel Tsai and Linda Nong}

University of Toronto, University of Guelph

Depression and obesity are widespread illnesses that are increasing in prevalence across the world. Suicide due to depression is a leading cause of death for youth and obesity appreciably increases the risk of other life-threatening medical conditions. This study aims to understand the correlation between these diseases on a global scale. The average BMI of a country was used as an indicator for the prevalence of obesity and depression levels were estimated as a percentage of the total population. Secondary data from 2015 was collected from the World Health Organization and a regression analysis was performed to determine the relationship between the prevalence of depression and the average BMI of a country. The results indicated a moderate, positive linear relationship. This was further supported by a low p-value (1.38e-8) and a categorical analysis which determined that, on average, countries with higher depression percentages had a higher mean BMI. In terms of modelling this relationship, a low R-squared value (16.5\%) suggested that the prevalence of depression cannot be directly used to model and predict the mean BMI for a given country. Future statistical models on this topic should include common risk factors to both obesity and depression, which include lifestyle habits, genetics, and social environment. An accurate model can help health officials identify populations at risk for developing high levels of depression or obesity based on demographic information, and target specific risk factors to decrease that risk.

Mentor: Mohammed Al Khaldi

Keywords: Depression, Obesity, BMI, Mental Health, Statistical modelling

\section{The Impact of the Canadian Tobacco and Vaping Products Act on e-Cigarette use and Perceived Risk in Adolescents}

Erica A. Li and Jeffrey Gutierrez

Western University, University of Toronto

Electronic-cigarettes or e-cigarettes have risen in 
popularity among adolescents in Canada. However, there is growing evidence of the harms associated with e-cigarette use. Recent studies show a rise in e-cigarette associated lung injuries. E-cigarettes use in adolescents may also increase the likelihood of starting use of conventional cigarettes. In response, Canada enacted the Tobacco and Vaping Products Act (TVPA) in 2018, which prohibited the sale of e-cigarettes to individuals under 18. However, this law increased the distribution and sale of e-cigarettes to individuals over 18. Presently, there is limited evidence on the impact of the TVPA on adolescents' use of e-cigarettes. The aim of this study is to assess adolescents' prevalence and perception of e-cigarette use before and after enactment of the TVPA. The Canadian Tobacco, Alcohol and Drugs Survey for 2017 and the Canadian Student Tobacco, Alcohol and Drugs Survey for 2015, 2017, and 2019 were obtained. Using RStudio, we conducted two-way ANOVA with Tukey's post hoc test. We reported the goodness of fit of each model through its multiple R-squared values and also conducted likelihood ratio tests. Results were presented as the mean with 95\% confidence intervals. We found that e-cigarette use was more prevalent in adolescents compared to adults prior to enactment of the TVPA. Interestingly, e-cigarette use in adolescents increased even after enactment of the TVPA, and this law did not impact adolescents' perceived risk of e-cigarette and conventional cigarette use. Furthermore, e-cigarette use in adolescents was highest in Saskatchewan and Newfoundland and lowest in Quebec and Ontario. Although the TVPA aimed to reduce e-cigarette use in adolescents, this study demonstrates that the TVPA in actuality generated the opposite effect. These findings highlight the need for improved public education and stricter e-cigarette sale regulations.

\section{Mentor: Hannen Abbas}

Keywords: e-cigarettes, cigarettes, adolescents, prevalence, perceived risk

\section{An Analysis of the COVID-19 Infodemic: The Irreversible Impact of US Public Figures on Sentiment towards Hydroxychloroquine}

\author{
Emily Chan, Ginah Choi, Kendrew Wong, and \\ Shirley Zeng
}

The University of British Columbia
An infodemic, described as an over-abundance of information that complicates the extraction of reliable information, poses a significant risk in spreading confusion and preventing protective measures from taking place during public health emergencies. As such, the impact of supporting and refuting actions and statements from US public figures and organizations, as well as relevant research, on public sentiment towards hydroxychloroquine were evaluated. Changes in public sentiment in relation to the top ten events yielding the highest Google News search results were measured by analyzing the sentiment of Twitter posts tagged with the hashtag or keyword "hydroxychloroquine" from February 24, 2020 to May 27th, 2020. Positive sentiment pertaining to hydroxychloroquine on Twitter always outnumbered negative sentiment throughout the pandemic. Statements made by Trump also gained significantly more attention as opposed to findings from research studies or statements made by reputable organizations such as the WHO or the FDA. One instance of a retracted statement concerning hydroxychloroquine efficacy made by the Department of Veterans Affairs had no statistical impact on sentiment ( $p$ adj> 0.05 Tukey HSD). Meanwhile, physician behaviour seems to be less susceptible to influence from public statements, as shown through decreases in hydroxychloroquine usage independent of major events. US public figures and research findings can influence public sentiment towards hydroxychloroquine; however, information relevant to hydroxychloroquine disclosed by Trump attracts a disproportionate amount of attention compared to other evidence-based informational sources, such as the WHO or FDA. Future contradictory statements cannot fully reverse public sentiment even if the initial statement is retracted. In a public health crisis, all statements and actions regarding contentious topics like hydroxychloroquine should be made with caution. Future research should be conducted using a trained model for sentiment analysis and an extended search query to validate the results of this study, or to study other public health infodemic issues.

Mentor: Anish Verma

Keywords: infodemic, sentiment, twitter, hydroxychloroquine, COVID-19

\section{Deep Learning Transcriptomic Model for}




\section{Prediction of Pan-Drug Chemotherapeutic Sensitivity}

Eddie Guo, Mehul Gupta, Pouria Torabi, and Sunand Kannappan

University of Alberta, University of Calgary

Emerging precision oncology studies have yet to generate a predictive biomarker that utilizes gene expression profiles to stratify tumours into similar pan-drug sensitivity profiles. This development would allow for identification of candidate drugs for treatments that maximize therapeutic response and minimize cytotoxic burden. As such, this study utilized cell line sensitivity and molecular profiling data to generate a combinatorial gene expression predictive biomarker, utilizing feature selection and a deep learning model. A pan-cancer cohort of cell-line gene expression data from Genomics of Drug Sensitivity in Cancer (GDSC) was clustered into two response groups. Cell line response groups showed a significant difference in pan-drug chemotherapeutic sensitivity. Due to the high dimensional nature of the microarray data, biologically agnostic feature selection was conducted to identify genes with the highest predictive value. The feature space was reduced to 300 genes, which functional profiling indicated was primarily enriched for the focal adhesion, ECM-receptor and proteoglycan interaction pathways. Using these selected genes, a deep learning neural network architecture was developed to predict response groups. Hyperparameter tuning of the deep learning model dictated a 4 hidden layer architecture with a predictive accuracy of $91.7 \%$. This validates the postulate that cell lines with similar gene expression profiles present similar pan-drug chemotherapeutic sensitivity, and it suggests the potential utility of similar combinatorial biomarkers for selection of potent candidate drugs.

Mentor: Daiva Nielsen

Keywords: clustering, neural network, transcriptomics, chemotherapeutic response, combinatorial biomarker, molecular profile, therapeutic sensitivity, cancer

\section{Combining Unsupervised and Supervised Learning Methods to Investigate Potential Country Development Factors Contributing to}

\section{COVID-19 Progression}

Feifei Li, Mian Li, iaxiong Jiao, and Jun Leng

University of Toronto

To have a deeper understanding of what might cause the various levels of COVID-19 transmission figure RO in different countries, we collected 144 country's GDP, population density, etc and their corresponding COVID-19 Ro value. By performing a Principal Component Analysis (PCA) and decision tree analysis, we were able to identify obesity rate and population as contributing factors correlated to COVID-19 transmissibility. Future research based on our preliminary results can focus on establishing the relationship between obesity and transmissibility of COVID-19 which may provide prospects in population health monitoring and disease control policy making.

\section{Mentor: Aliyah Dosani}

Keywords: COVID-19, PCA, Basic reproductive number, Unsupervised learning, Supervised learning, Decision tree, Linear regression, GDP

\section{Effectiveness of Diagnostic and Treatment Modalities for Breast and Prostate Cancer in Countries with Variable Levels of Development}

Roberto Fedrigo, Ehtan Haid, Adam Watkins, and Roberto Winata

University of British Columbia, Simon Fraser University, BC Cancer Research Institute, Vancouver

Chronic health conditions, such as cancer, have applied significant strain on health care systems in recent years, likely due to early detection and improved disease prognosis. Breast and prostate cancer both represent a considerable portion of chronic health conditions, as they are characterized by high incidence in the population and long survival. Despite the existence of successful methods for screening and diagnosing breast and prostate cancer, few studies have investigated the global impact of screening tools on patient survival rates and their economic repercussions. This paper aims to investigate how the implementation of diagnostic, therapeutic, and screening technology, for both breast and prostate cancer, impacts countries based on their level of 
development. Datasets were used to classify 194 nations based on their human development index (HDI). Quantities of diagnostic (MRI, CT, PET, gamma cameras) and therapeutic (linear accelerators and cobalt machines) modalities were correlated with relative mortality rates in each country, using the Pearson correlation. Strong, negative correlations were found between the number of machines and relative mortality rates, particularly for countries with an HDI in the top $25 \%$. Health care spending had a stronger correlation with the number of therapeutic, rather than diagnostic, machines ( $R 2=0.59$ and $R 2=0.20$ respectively). Overall, developed countries with high investments in diagnostic machinery, did not spend more in health care. Since it is well-known that early detection of cancer is associated with improved survival and quality of life, it can be asserted that investment in diagnostic machinery is beneficial from both a patient care and economic perspective. The widespread availability and success of national screening protocols was highlighted for breast cancer; indicating a possible unmet need for prostate cancer.

Mentor: Tesla La Touche

Keywords: cancer, early detection, public health

\section{Hospital Capacity Under COVID-19: Correlation between Infection-Hospital Bed Rate and Fatality Rate}

\author{
Gigi Chan, Crystal Shum, Eugene Yeung, and Lori \\ Jia
}

University of Toronto

During the 2020 COVID-19 pandemic, a major concern was that large numbers of people will contract the highly contagious disease in a short period of time and overwhelm local healthcare systems, resulting in high numbers of casualties as patients are not given adequate care. In light of this concern, physical distancing measures were introduced to 'flatten the curve' and maintain a manageable load for hospitals. The purpose of this paper is to test the relationship between the load on healthcare systems and the fatality rate of COVID-19 patients. Our data is drawn from 11 states in the United States. We conclude that strain on healthcare systems contributed to the increased fatality rate of COVID-19 patients. However, this effect must be evaluated together with other variables such as the demographic of people infected by COVID-19. Our findings offer empirical support to government physical distancing measures and show the need to further monitor the handling capacity of individual hospitals.

Mentor: Jason Steffener

Keywords: COVID-19, hospital capacity, fatality rate, physical distancing, pandemic

\section{A Public Health Perspective on whether the "New Normal" should be a Green One}

George Chen, Michael Gong, and Wu Jih Hsu

\section{University of British Columbia}

The COVID-19 pandemic has affected everyone, but during a climate emergency, how has the environment been impacted and what are the implications on public health? This project aims to investigate the environmental effects caused by the COVID-19 pandemic response and how the environmental implications could have on public health. Time series data of daily air pollutants ( $\mathrm{PM} 2.5, \mathrm{NO2}, \mathrm{CO}, \mathrm{SO} 2$, and $\mathrm{O} 3$ ) levels in 362 Chinese cities were obtained from the China Meteorological Administration and analyzed for significant change from January 1, 2020 to March 31, 2020. Multiple linear regression models were generated from American county-level air pollutant data and epidemiological data from various United States government agencies and health research institutes to investigate the relationship between the air pollutants and four chronic respiratory disease endpoints: tracheal, bronchus, and lung (TBL) cancer mortality rate, chronic obstructive pulmonary disease (COPD) mortality rate, COPD prevalence, and asthma related emergency department (ED) visits. The generated models were used to predict the change in endpoints caused by differences in pollutant levels before and after implementation of COVID-19 lockdown policies in five major cities in China. PM2.5, NO2, CO, and $\mathrm{SO} 2$ levels significantly decreased in most Chinese cities at around the same time as COVID-19 lockdown measures, indicating the significance human activity has on pollution. PM2.5 was the predominant contributor on all the models when predicting the endpoints. The models predicted that TBL mortality rate, 
COPD prevalence and mortality rate, and asthma related ED visits should decrease by $43.5 \%, 40.9 \%, 37.6 \%$, and $51.6 \%$, respectively. It would be prudent for governments to learn from how COVID-19 has revealed the human impact on the environment and the benefits cleaner air has on public health, therefore the new normal should be a green one.

Mentor: Christy Tomkins-Lane

Keywords: air pollution, multiple linear regression model, COVID-19, chronic respiratory diseases, public health

\section{Public Health in the Digital Age: Analyzing the Relationship Between the Influence of Twitter Social Media on Social Distancing Measures and COVID-19 Cases in the United States}

\section{Grace Huang, Jessie Wu, Nicholas Chung-Hun, and Varun Mokashi}

University of Western Ontario, University of Waterloo

The public health strategy to "flatten the curve" has recently become a widely trending phrase

across social media platforms as a means to share content related to COVID-19. Given today's digitally-focused society, we aimed to investigate the relationship between the Twitter tweet sentiments on critical COVID-19 social distancing policies in the United States (US) and the presence of COVID-19 cases. Using data sets from John Hopkins and Harvard University, we established the relationship between Twitter sentiment, as collected through natural language processing sentiment analysis techniques, and the change in COVID-19 cases in each US state over time. This uncovered potential to build a predictive growth model based on the Granger-Causality test examining how sentiments expressed through tweets would set a precedent for fluctuations in new COVID-19 cases. Additionally, we sought to understand the degree to which social media externally influences users to drive certain social distancing behaviour by building a linear regression model analyzing the relationship between likes and retweets of tweets expressing different sentiments and growth of cases. As a result, our conclusions suggest a three day time-lag relationship between social distancing tweet sentiments and fluctuations in COVID-19 cases. The conducted study was limited by virtue of varying state laws, discrepancies in state populations, and the exclusion of nonEnglish tweets, which elicit the need for multiple focused studies that more accurately explore these phenomena. In the future, iterations of the predictive growth model should account for more comprehensive tweet data sets in tandem to the geographical influence for the spread of the virus on a day-to-day timeline. Subsequent studies should also investigate leveraging social media as a tool to maintain or improve public health.

Mentor: Tom Oullette

Keywords: COVID-19, natural language processing, sentiment analysis, social distancing, Twitter

\section{Socioeconomic Determinants of Health and COVID-19 outcomes: A county level study of the United States}

Harshmeet Arora, Pranay Jain, and Sahil Mazmudar University of British Columbia

A range of medical, policy and socioeconomic factors have played a role in determining the spread of the coronavirus (COVID-19) in different communities. These factors vary largely across the United States, which presents a challenge to centralized planning and policy measures. A county-level analysis can provide deeper insight into the structural inequities that exist within the country and guide strategies that are better equipped to alleviate the public's hardships. Using publicly available county-level datasets, we identified key socio-economic factors influencing susceptibility of a population to COVID-19. We focused this study on 6 socioeconomic determinants of health: education, unemployment, income, rural-urban classification, access to hospital beds and access to primary care physicians. Education rate, rural-urban classification and unemployment were found to be moderately correlated with COVID cases per 1,000 people and deaths per 1,000 people. The regression analysis found education, unemployment, median household income, rural-urban classification and number of hospital beds to be statistically significant determinants of COVID outcomes. We use the derived insights to propose appropriate policy changes. We discuss how county level governments could improve health literacy using public engagement and minimizing misinformation. 
We recommend innovative public health policy reforms to finance better healthcare access for lower income groups. We also recommend a guaranteed employment policy as a preventative measure against future viruses. Finally, although access to healthcare through bed capacity and physicians per capita did not exhibit strong correlation with COVID-19 spread, strategies such as elective surgeries, rapid-response groups, and streamlined patient flow management show promise in dealing effectively with virus spread.

\section{Mentor: Aliyah Dosani}

Keywords: COVID-19, socio-economic, rural health, public health, health inequity, policy

\section{COVID-19 Mortality in Ontario Long-term Care Facilities by Service Type, Socioeconomic Status, and Population: An Exploratory Analysis}

Hani Choksi, Ruijia Zhang, and Sriprada Thallapalli

McMaster University

In light of the recent COVID-19 pandemic, several flaws in the Canadian healthcare system such as poor staffing and lack of resources have been exacerbated. This has been especially detrimental across Ontario's Long Term Care Facilities (LTCFs), where outbreaks have taken a toll on the already overworked staff and deaths in the vulnerable community. Here, we assess several factors that may make LTCFs more prone to an outbreak and to higher mortality, including socioeconomic status, local population, and service type. We use statistical measures such as box and whisker plots and regression models to assess the relationships between variables and Geographic Information Systems (GIS) to visualize data. Our results showed that while charitable homes for the aged (CHFAs) had the greatest percentage of outbreaks, for-profit LTCFs had the most severe outbreaks (i.e. highest mortality). By attempting to build a linear regression model for income and mortality, it was found that these two variables likely do not share a linear relationship as many linear regression model assumptions were not satisfied. The total population of all subdivisions of Ontario, when visualized on a map along with LTCFs and their COVID-19 status, illustrated that highly populated areas seemed to have the highest number of homes with an outbreak. In the future, these factors should be considered as Ontario continues to make improvements to ensure better quality and more consistent care in longterm care homes.

Mentor: Gloria Lim

Keywords: Long-Term Care Facilities, Ontario, Socioeconomic Status, COVID-19

\section{Analysis of COVID-19 Deaths, and the Influencing Factors}

\section{Heidi Rysan and Oluwatitomi Adebajo}

Western University

Coronavirus disease 2019 (COVID-19) has infected millions of people all over the world. A majority of the people who are more susceptible to the virus are old. In this study, old was defined as those who are 50 years and over. Research was centred around COVID-19 deaths among older populations from both Canada and the United States. Data for the two countries was collected from several sources and was analyzed using R and Python. Based on the results, it was determined that the US had a larger percentage of COVID-19 deaths. This could be due to the fact that Canada implemented lockdown procedures earlier, suggesting that early lockdowns could help prevent COVID-19 deaths during future waves of the virus. In addition, after performing multiple hypothesis tests, it was determined that females over 80 in both Canada and the US had the largest percentage of COVID-19 deaths which indicates that health policies to protect older, more vulnerable women are necessary. Furthermore, when specific states in the US were studied, housing was one of the factors thought to influence COVID-related deaths. There was a positive, moderately strong correlation between COVID-19 deaths and housing costs. Based on this, implementing affordable housing could help to reduce viral spread. Smoking and immunization were factors identified for Canadian COVIDrelated deaths. Over time, the number of Canadians aged 50-64 with influenza immunization has gone down. This highlights the importance of educating individuals on the necessity of vaccines, should there be a vaccine for COVID-19 developed in the future. Moreover, smokers are at a higher risk of dying from the virus, however further research on the relationship between COVID-19 deaths and smoking is 
required.

Mentor: Jenny Bicong Ge

Keywords: COVID-19, affordable housing, lockdown, vaccines

\section{Correlation between Alcohol Harm and Hospitalization for Traumatic Brain Injury (TBI)}

Janah Alameddine, Muneer Qazi, Alaa Alameddine, and Judy Alameddine

University of Waterloo, University of Ontario Institute of Technology

The intended purpose of this paper is to determine if Traumatic Brain Injury (TBI) correlates with high rates of alcohol harms in those between the ages 0 to 19 in the health regions of Ontario. With alcohol abuse being a significant public health concern in Ontario and over 77,000 teenagers reportedly being heavy drinkers, there is growing concern that TBI rates will increase among youth due to alcohol harm [1]. Datasets were collected from the Public Health Units (PHU) and Local Health Integration Networks (LHIN) of Ontario. The main objective of analysis of these datasets is to determine the rate of both incidents per capita in each health region in Ontario, and compare them. Analysis is performed through statistical hypothesis testing and comparing the difference in rates of both incidents. Through this method, no significant correlation was found between areas of alcohol harm and hospitalization due to TBI among youth in health regions of Ontario. While the absolute value of the difference in rates was mostly shown to be low, many health regions display a great range in the difference between the rates. As such, there is a weak correlation between the rates of hospitalization due to alcohol harm and TBI among youth in Ontario.

Mentor: Danielle Roy

Keywords: Traumatic Brain Injury, Alcohol Harm, Hospitalization

\section{Drug Use and Disease: Recreational Drug Use and COVID-19}

Josh Brauner, Kalum Ost, Karla Juego, and Stefan

\section{Gavrilluc}

\section{University of Calgary}

With Canada becoming the second country in the world to fully legalize recreational cannabis use [1] with the Cannabis Act [2], a landmark shift in recreational drug policy occurred. At the same time, the popularity of vaping began to rise [3], and with it returned the concern that recreational drug use may impact how diseases can spread. Conveniently, if tragically the COVID-19 pandemic of 2020 provides the perfect study space in which to assess such correlations. We aimed to determine how the usage of various drugs could affect the severity of COVID-19 within a community, alongside other suspected factors of COVID-19 mortality. To accomplish this, we compared the usage rate of various recreational drugs (cannabis, vapes, cigarettes, and alcohol) with that of COVID-19 mortality across North American and European countries (including Canada, the United States of America, and European Union members) using multiple linear regression, with a number of demographic factors in each population also being accounted for. Overall, the effect of recreational drug-use had no significant impact on COVID-19 mortality rates except for in Canada. However, conclusions drawn from the Canadian data set are limited by the small number of data points for each variable tested in the model. There may be other confounding factors that contribute to observed COVID-19 mortality which may have went unaccounted. Due to the size of our dataset and the ongoing nature of the pandemic, further investigation would be required to confirm these trends.

\section{Mentor: Zoya Retiwalla}

Keywords: COVID-19, cannabis, vaping, recreational drug use

\section{Assessment of Quality of Life on Organ Donor Registration Rates in Ontario Cities and Towns}

Jonathan Blumenthal, Yiyong Liu, Olivia MannDelany, and Shahir Haradwala

University of Toronto, Western University, McMaster Univsersity, Ryerson University

Organ donor registration rates in Canada still pale in comparison to those of other developed countries. Numerous initiatives have attempted to mitigate this issue, such as by 
raising awareness and simplifying the process of becoming a donor, yet they have resulted in little difference. Previous studies have identified certain demographics as less likely to register as donors. Education and income level distribution of a population were used as measures of quality of life to understand how these two socioeconomic factors can influence the organ donor registration rate of a population. Looking at eleven selected Ontario cities and towns and using publicly available data from the Government of Canada and Ontario's beadonor.ca, it was found that although education levels are not correlated with registration rates, income levels were positively correlated with registration rates.Meanwhile, there was no correlation found between education levels and registration rates. This data can be used to further target organ drives and to inform policymakers in Ontario.

Mentor: Vidhi Patel

Keywords: Organ Transplantation, Organ Donor, Quality of Life

\section{Correlating COVID-19 and Climate Policy: The Role of Foresight and Risk Assessment in Major Policy Issues}

Jacques Carmichael, Kitaro Jung, Jordan Legendre, and Julian Pucella

\section{McGill University}

Coronavirus Disease 2019, or COVID-19, is a disease affecting nearly every country worldwide [1]. Based on recommendations from public health experts, governments enforced regulations to control the outbreak of the virus. Global climate change is another issue affecting the world. In order to minimize the harmful effects of climate change, regulations are also enforced by governments. Government response to COVID-19 and climate change were correlated through the indices of the Oxford Coronavirus Government Response Tracker (OxCGRT) and Germanwatch's Climate Change Performance Index (CCPI) respectively. Additionally, two other metrics for coronavirus response were correlated with the CCPI. For the three correlations, the root mean square values were of $0.0013,0.0076$, and 0.0001 , illustrating no substantial correlation. It was proposed that greater factors in the decision to impose COVID-19 measures were decreased incentives for international trade, the observation of severe health outcomes in other countries, and pressure to do so from growing international consensus. Resulting insights into climate policy are that action will likely not follow early scientific evidence, but rather drastic health outcomes elsewhere in the world and a rapid growth of international consensus. Potential risks of this strategy are that when climate change becomes so apparent, it may be too late to avoid the worst outcomes.

Mentor: Jason Steffener

Keywords: COVID-19, Coronavirus, Climate Change, Climate Action

\section{Sedentary Behaviour and Heart Disease}

Jessica Chang, Rachel Ombok, and Abrar Alam

of British Columbia, New York University, Ryerson University

Due to an increase in the forms of electronic entertainment being offered to consumers, there has been an increase in sedentary behaviours, activities that involve little to no movement, such as watching the television. As the age of technology has become especially prevalent for adolescents, so have the risks associated with sedentary behaviours. This study examined the link between increased sedentary behaviour and an increased risk for cardiac disease. The databases used to conduct the study included: The Government of Canada Open Data Source, where relevant data was obtained to be analyzed via SAS software.

Mentor: Sanjai Arumugam Jaganmohan

Keywords: healthcare, heart disease, physical inactivity, sedentary behaviour

\section{An Analysis Of COVID-19 Data to Identify Key Social and Policy Factors Affecting its Progression and Forecast Future Trends}

\section{Jia Cui, Yuzhou Liao, Qiaochu Zhang, and Ophelia Zhao}

University of Waterloo

COVID-19 is no doubt so far the biggest public health 
crisis in this century. Looking throughout history, it is also viewed as one of most influential pandemics to peoples daily lives. By studying its current data and predicting future trends, we believe it can provide valuable insights to develop better coping strategies with similar future crises, including a possible second waves of COVID-19. We used the datasets provided by the website OurWorldinData and the University of Oxford. We started off by investigating the correlation between several factors and the progression of this pandemic, using logistic and exponential regression. After the detailed analysis of each factor, we progressed by feeding these contributing factors into a neural network, and predicted the turning point for countries currently at the high growth stage. In the end, we forecast the confirmed cases in 12 countries using the ARIMA model and BSTS model. We discovered that the lockdown policy, healthcare system availability, mobility information, population density, and demographic factors all have a correlation with the progression of COVID-19. As for predictions, we concluded that the new cases will start to decrease by end of June; and future trends for main countries are forecasted respectively by various models.

Mentor: Priyanka Gogna

\section{Home Alone: The Impact of Solitary Living on Physiological Health}

Jessie Deng, Sophie M.A Effing, Hung-Yu H. Lee, and James A. Sousa

\section{Queen's University, University of British Columbia}

Many studies have shown that social isolation has a detrimental impact on mental health, one such study citing a higher purchase rate of antidepressants by individuals who live alone. In addition to the mental health component, other studies have shown that living alone increases the risk of developing certain health conditions and affects all-cause mortality. This study explores the impact of living alone on physiological health using physiology, demographic, and medical condition data compiled from the 2017-2018 National Health and Nutrition Examination Survey (NHANES). While the survey included 9254 Americans of all ages, it was filtered to only include participants ages $21-80$, resulting in a sample size of 5493 . We hypothesized that living alone produces a decrease in overall physiological health and evaluated this using the Pearson correlation coefficient, Chi-Squared test, and Analysis of Variance (ANOVA) in RStudio. The findings provide support to our hypothesis and suggest that living alone increases the likelihood of developing high blood pressure and various liver conditions. While the correlation data for other conditions also showed a positive correlation between living alone and risk of developing a disease, those results were not statistically significant, potentially due to the sample size being too small for those specific conditions. Other limitations of our analysis include the grouping of liver conditions as one variable instead of by individual disease and the potential confound of pet ownership.

\section{Mentor: Kamal Ben}

Keywords: Physiological Health, Living Alone, Social Isolation, Liver Condition, Cardiovascular Health, Systolic Blood Pressure

\section{A Predictive Model of COVID-19 Case Rates Based on Social Determinants of Health in Public Health Units of Ontario}

Jiin Kim and Zara Ahmad

\section{University of Toronto}

Coronavirus disease 2019 (COVID-19) was classified as a pandemic by the World Health Organization (WHO) and Canada was not exempt from its reach. One might assume that COVID-19 cases might differ between two Canadian provinces due to distinctive government restrictions, however, a question arises when the rate of COVID-19 cases show significant differences within a province, especially if two areas are adjacent to each other. This study aims to examine the potential social determinants of health (SDH) that might be contributing to the differences in COVID-19 cases per 100000 between Ontario Public Health Units. Possible SDH were extracted by examining the 2016 Canadian census rates for 29 health units, and a model was created based on SDH that could accurately predict the number of COVID-19 case rates in different Ontario regions. This study identified 14 independent variables that together projected COVID-19 case rates per 100000 with a great level of prediction $(\mathrm{R} 2=$ $.800)$. These variables were: 65 years and over, households 
with 1 person, households with 5 or more persons, census family without children, census family with children, nonimmigrants, immigrants who arrived before the age of 5 , third generation or more immigrants, Asian immigrants, Aboriginal identity, total visible minority, trades; transport and equipment operators and related occupations, commute duration of 60 minutes and over, and commute duration of less than 15 minutes. The present model was able to predict COVID-19 case rates with various SDH. This model could be used to predict the effect of future outbreaks in Ontario, and thus be helpful in guiding public health policies for infectioncontrol. Future study should examine other provinces in Canada, or other countries, to see what other SDH patterns emerge and create a predictive model which might provide profound insights to reduce the spread of infectious diseases.

Mentor: Mohammad El-Hajj

Keywords: COVID-19, Social determinants of health, Machine learning, Model

\section{Contribution of Electronic Cigarettes to Tuberculosis Incidence Rates in Canada}

\section{Jessica McDowell and Izzar Linares}

\section{University of Toronto}

Cigarette smoking is associated with increased risk of tuberculosis (TB) infection, active TB disease, relapse following treatment and death from TB; however, there has been little research examining whether e-cigarette use increases the risk of TB infection among Canadians. This paper aims to determine if the prevalence of e-cigarette use (ever use and past 30-day use) among Canadians correlates with the incidence rate of TB within Canada. The research presented in this paper is important for Canadians of all ages because e-cigarettes have increased rapidly in recent years, with several studies reporting higher rates of e-cigarettes use among Canadian teens, and TB remains the world's most deadly infectious disease. E-cigarette statistical data was collected from Statistics Canada's 2013, 2015 and 2017 Canadian and Tobacco, Alcohol, and Drugs Survey. TB statistical data was collected from the Canada Communicable Disease Report (CCDR) Tuberculosis in Canada, 2017. Pearson's correlation test was used to investigate the correlation between TB incidence rate and e-cigarette use (ever use and past 30-day use), and the coefficient of determination (R2) was used to evaluate the quality of fit of a model on data. 2013, 2015, and 2017 data are the most recent years for which data is accessible. Health Canada's Canadian Tobacco, Alcohol and Drugs Survey did not include questions on e-cigarette use before 2013. The statistical data analysis performed throughout this report does not support the hypothesis that there is a positive correlation between the prevalence of e-cigarette use and TB incidence rates. The evidence for the absence of a correlation may be due to the lack of statistical data available for the prevalence of ever use and past 30-day use of e-cigarettes among Canadians. Health Canada's Canadian Tobacco, Alcohol and Drugs Survey did not include questions on e-cigarette use before 2013. Future studies should continue to analyze the correlation between e-cigarette use and TB incidence rates in Canada. The sharp increase in the prevalence of ever use and past 30-day use among Canadian youth and young adults highlights the compelling need to learn more about this evolving class of products and determine whether a correlation between e-cigarettes and TB incidence rates exists, which would ultimately have a profound effect on health - requiring health impact assessments for polices, programs, and projects.

Mentor: Michael Dorrington

Keywords: E-cigarettes, tuberculosis, mortality, incidence, nicotine

\section{Correlation between Antibiotic Resistance and Consumption of Amoxicillin in Canada}

Jennifer Wang, Jocelyn Yao, Landy Xu, and Selina Huang

\section{University of Waterloo}

Antibiotics play a significant role in reducing infections caused by bacteria. However, antibiotics resistance can happen at any stage of life in any country. This paper aims to provide insights into the relationship between consumption of Amoxicillin and its respective antibiotic resistance rate. To investigate this aim, data sets from Canadian Antimicrobial Resistance Surveillance System (CARSS) and Canadian Antimicrobial Resistance Alliance (CARA) from 2010 to 
2017 are being analyzed along with two common bacterial disease: Streptococcus pneumonia and Escherichia coli infections. Excel VBA is used to reorganize the data and R is used to conduct the Pearson correlation test. In addition, Analysis of variation (ANOVA Table) was used to determine whether there are interactions between different levels of resistant or not and this can lead to a more accurate model. Through these analysis, we found that Amoxicillin cavalanic acid has different antibiotics resistance influences on these two bacterial diseases. In conclusion, there are no distinct correlation between the consumption of Amoxicillin and its antibiotic resistance when analyzed respect to the two selected bacterial disease. Future research can be done to investigate the mechanism of antibiotics when used against different bacterial disease as the mechanism and performance of the antibiotic agent can vary from bacterium to bacterium. Since Amoxicillin is one of the most common prescribed antibiotics, we can assume that other antibiotics may have similar results. Thus, same antibiotic could potentially have different resistance effects on different bacterial diseases. Gathering more data for different antibiotics used on different bacterial disease can help government in adjusting prescription policies and promote appropriate use of antibiotics. For individuals in concern with bacterial disease, it is recommended to see professionals regarding the use of antibiotics and the possibility of carrying resistant bacteria as it can have a large impact on one's medical treatment.

\section{Mentor: Matineh Panah}

Keywords: Amoxicillin, antibiotics resistance, bacterial disease, Streptococcus pneumonia, Escherichia coli

\section{Leveraging Machine Learning Methods to Predict COVID-19 Vulnerability in U.S. Counties Based on Socioeconomic Factors}

Katharine Emily Lee, Cynthia Denise Lo, William Ren $\mathrm{Xu}$, and Robert Ye

Johns Hopkins University

As COVID-19 gained pandemic status, the number of confirmed cases in the United States surpassed that of all other countries. Although the virus spread throughout the U.S., not all areas were affected equally. This retrospective study aims to explore these inequalities through

pre-pandemic socioeconomic characteristics by creating a predictive model for COVID-19 vulnerability at the county level. A total of 103 features of socioeconomic data for 2734 U.S. counties (out of a total of 3007) were sourced from various online databases such as the U.S. Census Bureau, the U.S. Department of Agriculture, and the Association of American Medical Colleges. Additionally, to quantify each county's COVID-19 situation, we defined two custom measures: severity, representing the number of COVID-19 cases per county population, and lethality, representing the number of COVID-19 deaths per confirmed county cases. Machine learning classification algorithms including Random Forest, Neural Network and Support Vector Machine were then used to predict the severity and lethality of US counties. Our analysis revealed that we can accurately predict a county's lethality score with about $55 \%$ accuracy, and severity with about 39\% accuracy. The moderate success in predicting lethality has provided insight into the existence of a hidden link between regional socioeconomic characteristics and COVID-19 susceptibility. In addition, the difficulties our model had with severity prediction solely based on pre-pandemic conditions supports the notion that region specific pandemic policies play an important role in successfully mitigating this public health crisis. Further studies are recommended to incorporate more robust data and to devote more time to optimizing algorithms.

Mentor: Christy Tomkins-Lane

Keywords: COVID-19 Vulnerability, Machine Learning, Predictive Model

\section{Exploring the Correlation between the Spread of COVID-19 and Air Temperature via Time Series Forecasting with Recurrent Neural Networks}

Kate Kim, Kattly Li, Hunter Murdoch, and Logan Ralston

\section{McGill University}

With the rapid spread of COVID-19, many factors are being studied in an effort to reduce the rate of its transmission. One commonly studied factor in respiratory viruses is 
seasonality - the idea that diseases are more active in certain environments and climates. Considering that the ongoing pandemic has more significantly impacted drier and cooler areas than the tropics, this study aims to investigate the role air temperature plays in the spread of COVID-19. The main method of analysis was to train two Long Short-Term Memory (LSTM) models with data from John Hopkins University and the National Oceanic and Atmospheric Administration (NOAA). The data included the new number of cases per day and the daily maximum and minimum temperature for each US county. The first model was trained using case data while the second model was trained using both case and temperature data. This analysis found that there was no significant statistical difference in the error between the models. Thus, it can be concluded that temperature alone has little effect on the spread of COVID-19. Future studies that investigate the seasonality of COVID-19 should strive to determine if other climate factors such as humidity and wind speed in combination with the air temperature will have an impact.

Mentor: Gloria Lim

Keywords: COVID-19, temperature, seasonality

\section{Efficiency of GPS Tracking in Reducing Transmission of COVID-19}

Louis Park, Rebecca Preyra, Aryana Zarandi, Alicia Morielli

\section{McMaster University, Western University}

This study aims to investigate the relationship between the GPS tracking status of a country and the number of days required for COVID-19 to reach a reproduction number of 0.5. The variables of interest are a country's GPS tracking status and its Plateau Date. An ANOVA test was performed to examine this relationship. A t-test was later conducted to determine whether a significant statistical difference between the Plateau Date of "Tracking" countries and "No Tracking" countries existed. A $95 \%$ confidence interval was used to make a predictive model. Through this model, a significant relationship was found: countries that adopted GPS tracking tended to reach a reproductive number of 0.5 , thus having a non-zero value for the Plateau Date. Comparatively,
"No Tracking" countries did not show a plateau in their epidemiological curve and were assigned a value of zero for Plateau Date. The significance of the p-values and the predictive model indicated that countries with GPS tracking achieved a 0.5 reproductive number for the virus in about 12 17 days since the peak. It was also discovered that universal healthcare, higher democracy score, and higher average age also positively impact a country's Plateau Date. As the pandemic onset time increases and more data is collected on the reproductive number by country, this relationship will be solidified.

Mentor: Matineh Panah

Keywords: COVID-19, Privacy, Geo-tracking, GPS

\section{Analysis of Relative Humidity and Mobility Rates on COVID-19 Transmission through Machine Learning Techniques}

Lakshay Sood, Michael Steinberg, Ritvik Ramakrishnan, Viraj Kacker

Johns Hopkins University, Northeastern University, Georgia Institute of Technology

This study aims to further analyze the recent claims made regarding humidity and mobility affect on COVID-19 case rate. Since the United States has slowly begun reopening, many states have not experienced as high of an incline as predicted, indicating other factors at play that could potentially affect COVID-19 transmission. To investigate this problem, this investigation employs a Machine Learning technique known as Feature Importance to uncover what other potential factors could be impacting transmission rate. Our feature importance model was able to identify the factor of relative humidity to account for $40 \%$ variation of COVID-19 cases, where as a combined social distancing effort accounts for $48 \%$ of variation. Our investigation was able to provide further supplemental analysis to the claims of humidity being a driving force in COVID-19 transmission.

Mentor: Marichelle Leclair

Low-Infection Re-open Sequence of Canada 


\section{Using GLEaM Model}

\section{Leni Wen, Weixi Zhuo, and Estella Liu}

University of Waterloo

The COVID-19 pandemic has pressed the pause button for almost every economic entity in the world and Canada is no exception. While the curve has been flattened by the joint effort of the people, the decision to re-open Canada requires caution against another wave of the epidemic. This is a decision that can only be harder than the initial lock down order and so far there has not been a clear path for the academia and the government. This paper aims to explore a reasonable re-open sequence of Canadian cities that can minimize the infection number while balancing the economic trade off. As this problem is of paramount importance, numerous statistical simulations have been conducted using the Global Epidemic and Mobility (GLEaM) model to forecast the outlooks of different re-open decisions. The GLEaM model has been praised for its accuracy in infectious disease modeling and flexibility in the scope of spatial data. A viable model also requires fine tuning of its parameters. We trained our GLEaM model with the 125-day Canadian COVID-19 time series along with population-distance weighted mobility matrix, then utilized Bayesian Optimisation to enhance forecasting abilities. Our GLEaM is able to help building a policy model that eventually suggests policy makers to reopen cities with lower populations before cities with high populations. The policy model can provide detailed reopening date of each region.

Mentor: Rakhi Konar

Keywords: GLEaM model, statistical simulation, Bayesian optimisation, public health policy

Comparing COVID-19 and Seasonal Influenza Cases to Predict the Vulnerability of At Risk Populations During the Probable COVID-19 Second Wave in Canada

Mustafa Alzahran, Jamila Sargean, Maria La Rosa, and Amalia Secenj

Laurentian University

Data on the COVID-19 cases of Canada during the pandemic was analyzed in comparison to seasonal influenza to predict the vulnerability of at risk populations during the probable second wave of COVID-19 in Canada. Population exposure levels and health effect values observed during seasonal influenza compared to that of the COVID-19 pandemic, will allow us to understand the severity of the second wave. Furthermore, using these results to implement policies that alleviate the threats to these populations. The information was gathered from databases such as the Government Canada, Health Research Data Resources, and the National Centre for Health Statistics. The focus of the analysis was to identify the trends of infection in the seasonal influenza and COVID-19 cases and identifying at risk populations. To analyze, a t-test and f-test was conducted on Microsoft Office Excel. In addition to these methods, HTML and CSS was utilized to create a visualization of the data for comparison. The results revealed that there is no significant difference between COVID-19 cases and influenza indicating that influenza may magnify this nuisance.

\section{Mentor: Omar Shafiq}

Keywords: COVID-19, Influenza, Pandemic, Canada, HTML, CSS

\section{Correlation Relationships of GDP, College Graduate Proportion and Population Size Versus COVID-19 Spread in America}

Michael Guo, Jason Zhao, and Serena Yeung

University of British Columbia

This paper aims to determine the trends and relationships between population size, proportion of college graduates, and GDP per capita of 48 states against the spread of COVID-19 caused by SARS-CoV-2. The number of days between the 100 th to 2000th case reported in each state were taken as the output variable to locate any trends in patterns between the three predictor variables to identify the nature and strength of their correlation with the initial virus transmission. This was done through a multiple regression analysis supported by plotting each variable separately against the output variable. Data was collected from the 2019 US Census, the Bureau of Economic Analysis (BEA), and the United States Department of Agriculture (USDA). The regression model including all 
three predictor variables has an adjusted R-squared value of 0.6351 , while the model excluding the college graduate proportion variable has an increased adjusted R-squared value of 0.6409 , which translates to a moderate strength even in the fit of the better model. This suggests that regional wealth and education among people have very little influence on disease spread, with population size being the only variable influencing the spread considerably. Consequently, further social distancing policies should be affirmed to reduce numbers of people in population-heavy areas. With further data collected on COVID-19 in the future, revisiting this discussion while emphasizing different demographics as well as considering other factors like transportation may yield more significant results.

\section{Mentor: Priyanka Gogna}

Keywords: COVID-19, GDP, College Graduation Proportion, US State Population

\section{Correlation Between the Use of User-Image- Based Social Media and Eating Disorder Rates}

\section{May Jagodkin, Joanna Roy, Sarina Xi, and Shiqi Xu}

\section{University of Toronto}

Recently, researchers have identified elevated social media use as a potential risk-factor for the development of eating disorders due to its impact on users' body image [1] [2] [3] [4] [5]. Namely, the use of user-image-based platformsthose containing photos of the users themselves-can lead to hyper-vigilance of one's body, perceived flaws and a fixation on posted "likes" and comments pertaining to visual appearance [6]. This paper examines the correlation between use of userimage-based social media platforms and eating disorder rates in Canada, the United States, and the United Kingdom, investigated using Python 3 libraries with data retrieved from $\mathrm{CIHI}, \mathrm{NHS}$, and Statisca. The analysis is performed using linear regression models that compare the age distribution of social media usage to that of the age distribution of eating disorder rates in each country. The root mean square value is used to determine whether a linear relationship exists. These models expose a potential correlation between eating disorders and the use of certain user-image-based platforms such as Snapchat and Instagram. However, more detailed data is required to illustrate significant correlation between the eating disorders and the use of user-image-based platforms. As such, further investigation is required to fully understand and examine the relationships.

Mentor: Mohammed Mamun

Keywords: social media, self-image, Canada, United States, United Kingdom, eating disorder

\section{Diabetic Prevalence Among Racial Groups and Their Susceptibility to Pandemics: Mitigating Future Harm}

\section{Michael Wang, Shubh Fageria, Abdalla Abdelhady, and Geedhanjali Vivekanandan}

\section{Western University}

The coronavirus (COVID-19) pandemic has placed great strain on financial and healthcare systems around the world. Since the start of the COVID-19 outbreak, over six million cases and over 350 thousand deaths have been reported worldwide. The United States (U.S.) stands atop of the COVID-19 charts with almost a third of the world's total cases and deaths. However, this pandemic is not the first outbreak of an infectious disease and will likely not be the last. Therefore, it is crucial to identify areas of improvement and proactive initiatives that would better the outcome of future outbreaks, especially for at-risk racial groups who are disproportionately affected by health issues. This report examines the differential effect of the COVID-19 pandemic on racial groups in the U.S. using publicly available data and statistical analysis tools such as R. It also highlights the relationship between COVID-19 deaths in racial groups and the prevalence of diabetes. In African American populations, racial COVID-19 mortality rates were found to increase with respective population sizes 2.5 times faster than in White populations. Also, the racial distribution of total COVID-19 deaths in African American populations was found to have a positive correlation with the diabetes prevalence across states at a rate of $4.8 \%$. Using a cost-benefit analysis, we showed that extending the National Diabetes Prevention Program to enroll all prediabetic, African American, Medicaid beneficiaries in the state of Louisiana may prevent more than 18,000 individuals from developing diabetes just three years 
after the implementation of the program. Also, the financial benefits of the program would outweigh the costs less than seven years post implementation. Our results highlight the benefits of using proactive preventative health measures to protect at-risk racial groups in the U.S. from future pandemics.

\section{Mentor: Tesla La Touche}

Keywords: race, diabetes, COVID-19, cost-benefit analysis, Medicaid

\section{Get Them Out: SEIR Models to Predict COVID-19 Evolution in Canadian Prisons}

Noémie Benoit, Zoé Benoit, Andréanne Boulanger, and Thomas Larente

Université du Québec à Montréal, Université de Montréal

Since the novel coronavirus outbreak was officially declared a pandemic by the WHO in March, international efforts have been devoted to mitigate its consequences. It is known that COVID-19 disproportionately weighs on marginalized populations, but its repercussions on the incarcerated population remain largely overlooked. In this context, the present study aims to predict the transmission dynamics of COVID-19 and its impact on 58 Canadian detention centres. The population of 17 Quebec correctional facilities was retrieved from the 2017-2018 prospective analysis of the Quebec prison population, while the capacity of 41 Canadian correctional institutions was used as an estimate of the federal prison population. Simple stochastic SEIR models were implemented to evaluate different scenarios of COVID-19 transmission in prison settings. We predicted that in a pessimistic scenario $(R O=14.8)$, the totality of inmates in federal and provincial detention centres would be infected in an average of 36.83 days, while in an optimistic scenario ( $R 0=1.78$ ), $50.7 \%$ of the population under study would have been infected by the virus 200 days after first exposure. In realistic scenarios, all inmates in the provincial correctional facilities ( $R 0=6.85$ ) would be infected in an average of 79.35 days, while $98.2 \%$ of the inmates in the federal correctional institutions ( $R O=4.1)$ would have been infected after 200 days. We believe that to avoid pessimistic and realistic scenarios of COVID-19 transmission in Canadian detention centres, as well as to protect vulnerable inmates, a process of selective release of at-risk inmates should be initiated. To our knowledge, this study is the first to model potential outbreaks in Canadian detention centres. We hope future research will consider addressing the same issue when more accurate data becomes available on both Canadian incarcerated population and COVID-19's epidemiology.

Mentor: Vishal Satyavinayak Purohit

Keywords: SEIR model, COVID-19, vulnerable populations, prison, prison health

\section{Evaluating COVID-19 Risks and Containment Measures}

Nicholas Lupul, Tian Su, Nirudika Velupillai, and Yaying Zhong

Macewan Universty

The purpose of this study was to find a model that would effectively predict how people perceived the threat of COVID-19 for themselves and others. The dataset, compiled by Dr.Adrian Gadient-Brügger, consists of 1272 observations and on 86 variables each variable contain one question. Based on the correlation matrix and plot, 9 variables were considered for this analysis, in addition to such as age, children, gender, education and work. Classification trees were created to see what factors were most influential. Multinomial logistic regression models were fitted and backward elimination was used to find the best model. Due to the imbalanced nature of the response variables under consideration, undersampling was used to create subsets of the train set, which were then subjected to multinomial logistic regression modelling and backwards elimination respectively. The final models were chosen based on Akaike's Information Criteria (AIC), precision and sensitivity. Results suggest that the extent to which respondents felt that COVID-19 would be a threat for themselves was influenced by the government's actions to protect the people, their age, how significant keeping distance in transmitting fewer diseases was to them, how safe one felt keeping their distance and at what rate they followed the recommendation to stay home. Perceived threat of COVID-19 for loved ones/others was influenced by to what extent respondents thought the health care system would become overloaded, their respective age, and how significant keeping 
social distance was to them.

Mentor: Mohammed Al Khaldi

Keywords: Threat, COVID-19, Mild, Severe, Distance

\section{Efficacy of Canadian Landmark Governmental Health Policies Against the Spread of COVID-19}

\section{Noah Varghese, Raj Mukhopadhyay, Henry Say,} and Robbie Stewart

\section{University of Western Ontario}

The onset of a pandemic such as COVID-19 calls for swift and effective implementation of health policies to slow the spread of the disease. These measures are introduced by governments at municipal, provincial, and nationwide level. It is imperative to evaluate these measures for their efficacy in mitigating the spread and flattening the curve. This information will be crucial to better prepare the general population for future pandemics of this magnitude, by highlighting the types of measures that are worth implementing and which ones may need re-evaluation or refinement. Our data was obtained from the Government of Canada's public health infobase and included the number of confirmed COVID-19 cases in Canada by province and by date. The increase in growth of infection rates for confirmed positive COVID-19 cases were studied in the two weeks prior and the two weeks after the implementation of select nation wide policies or recommendations (by highly ranked public health officials). Analysis was done by calculating the average percent growth, or the rate at which the infection (measured as the number of confirmed cases for each day) was accelerating in each province across the two-week time frames of study in Microsoft Excel. This data was then transferred to R-Studio and pairwise t-tests were performed for each of the landmark policies/recommendations of study. Across all three of the policies/recommendations that were analyzed in this study, there was a significant decrease in the daily growth of infection rates, with Alberta as the only exception for one of the policies.

Mentor: Kristen Malik

Keywords: measures, COVID-19, policy

\section{Factors Influencing Blood Pressure of Individuals Residing in Canada Aged 65 and Older}

\section{Oscar Zhen, Jacob Lee, and Dustin Li}

\section{Simon Fraser University}

This paper aims to identify those factors positively correlate with high blood pressure to provide particular areas for future research and solutions to focus on. The target population is individuals of ages 65 and older and residing in Canada. Blood pressure levels reported by male and female Canadians is contrasted with the number of individuals reporting mood disorders, daily smoking habits, and heavy drinking habits. The findings indicate a positive correlation between mood disorders, daily smoking habits, and high pressure in males. No positive correlations could be identified for females.

\section{Mentor: Nayaar Islam}

Keywords: high blood pressure, senior, 65 and older, heavy drinking, mood disorder, current smoker

\section{Dissemination of Canadian Provincial Public Health Policy about COVID-19 on Instagram and Twitter}

\section{Payton Mackwood, Hannah Illing, and Emilie Kaye}

University of British Columbia

This paper aims to determine the influence social media has on the spread of misinformation about COVID-19, as well as to compare the techniques employed by different provinces. Previous studies suggest that governments' use of social media can be used to prevent the spread of rumours and misinformation on social media. Using a thematic grounded theory approach, action statements were analyzed from each province's Twitter and Instagram account. Furthermore, by looking at the top ten comments per post it can be concluded whether the government's post had a positive or negative influence on society. The number of action statements used on Twitter was correlated to the number of people leaving their house as seen using "Google COVID-19 Community Mobility Reports." By comparing the number of COVID-19 cases with the number of posts per Instagram there is not 
a clear correlation. Future studies should examine private accounts as well as more social media platforms.

Mentor: Marichelle Leclair

Keywords: social media, COVID-19, government media presence, misinformation

\section{Determining Factors That Are Negatively Associated With Personal COVID-19 Curve- Flattening Behaviors in the United States}

\section{Rehab El-Hajj, Sarah Davis, and Raymond Otieno \\ University of Alberta}

COVID-19 has arguably impacted every dimension of social living - be that employment, schooling, healthcare or recreational activities. In a matter of months, businesses have shut down and the workforce and schools have been redirected to online work in many regions of the world. One key element of the North American pandemic response has been the emphasis that the spread or prevention of the pandemic is largely dependent on the measures taken by residents of any region. As such, our central research question focuses on outlining the factors that determine if an individual is less likely to take this pandemic seriously (i.e. is taking fewer measures to prevent the spread of COVID-19). We have analyzed the results of a U.S. wide COVID-impact survey using random forest classification (RFC) to associate individual factors such as employment status, household income, self-reported state of mental health, gender, age, family size, etc. to measures taken against the pandemic such as washing/sanitizing hands, staying six-feet away from anyone outside the home, cancelling recreational activities, etc. Our results indicate that the top three influential factors for determining whether someone will take the pandemic seriously are household size, the number of adults living in one household and the health of the respondent (poor to excellent). Using these insights, we narrowed the search and used association rules to determine key combinations of features that may lead to an apathetic response to a global pandemic in U.S. citizens, such as lower income households. Overall, this research allows us to explore the complexities of personal health decisions to help guide public health decision-making related to the COVID-19 pandemic.
Mentor: Ravi Kiran Selvam

Keywords: COVID-19, 2019-nCoV, coronavirus, virus, safety, protocols, distancing, prevention, demographics, machine learning, data mining, analytics, random forest, association rules

\section{An Investigation into the Economic Determinants of Suicide Rates in the US}

Ruyan Geng, Jia Yi Ong, Muhammad Ali Sajid, and Francisco Sandoval Macias

University of Toronto

We study how suicide rates respond to economic determinants such as unemployment rates and median salary using data from the United States. Our project is inspired by threats of an imminent recession and our concern regarding its secondary health outcomes. We seek to investigate a potential need for investment in preventative measures by governments. This can be in the form of mental health support, counselling, and greater individual support for those in the labor force. Our aim is to avoid a complete shift of governmental focus away from health issues once the pandemic is under control, as there are risks associated with the economic downturn that will surely follow. To study our problem, we extract data from the Centers for Disease Control and Prevention (CDC) online database. Some of the variables in our master dataset include country-level data on suicide rates, unemployment rate, median salary, and drug-induced deaths. We conduct a regression analysis based on a generalized linear mixed model (GLMM). We show that unemployment does not impact suicide rates directly. However, there is a significant positive correlation between suicide rate and salary. There is also a highly significant, strongly positive correlation between suicide rates, druginduced deaths and alcohol-induced deaths.

Mentor: Zoya Retiwalla

Keywords: Suicide rate, recession, mortality

\section{Correlation Between Population Density and COVID-19 Impact}




\section{Raymond Romaniuk}

\section{Brock University}

The Coronavirus Disease 2019 (COVID-19) has significantly impacted individuals across the planet. This paper aims to determine which factors within our society had an impact on the mortality rate in areas like New York City and Italy, and why these two locations were dramatically affected. COVID-19 data was extracted from the Johns Hopkins Coronavirus Map along with provincial/state level data from Statistics Canada, the US Bureau of Economic Analysis, the US Census Bureau, Stats America and the Centers for Disease Control and Prevention. Python was used to manipulate the data and position it in an optimal format for analysis. Linear regression models were then employed, in SAS, to determine how smoking[1][2], obesity rate[3][4], age[5][6], household size[5][7], population density[8][9] and GDP per capita[10][11] affected the COVID-19 outbreak in a given area. The models were tested with different data sets to determine the optimal model for predicting the ramifications of COVID-19 outbreaks in certain areas. Principal Component Analysis was also used in SAS to explore the correlation between the different variables. Aside from statistical analysis SAS was used to plot the linear regression model and display $95 \%$ conifidence and prediction limits. Power BI was then used for supplementary visualizations. Upon analysis of the results it was apparent that population density had the greatest impact on the state of a pandemic within a given city. This finding helps explain why a city like New York was overwhelmed with cases. Another interesting discovery was the relationships between population density and obesity rate. Intuitively, one would think that a higher obesity rate in an area would cause for a higher percentage of the population to fall ill, however this is not the case. It turns out that the states with higher population density actually had some of the lowest obesity rates and these states had a higher percentage of mortality than those with high obesity rates and lower population density. The population density issue has come to the forefront during the current pandemic, but with that issue a solution has also been created and that is the surge of remote work.

\section{Mentor: Mohammad El-Hajj}

Keywords: Coronavirus Disease 2019, COVID-19, Pandemic, New York

\section{Examining the Impact of Sociodemographic Features in the USA at a State Level on Reproduction Number Estimates for COVID-19}

\section{Rachel Wong, Christina Seo, Sheree Zhang, and} Litong Zheng

University of Toronto

As the COVID-19 pandemic affects billions of people across the world, it is crucial for researchers and policy makers to have a proper understanding of how quickly the virus is being transmitted. The basic reproduction number, Ro, is the most commonly used variable to measure this spread. While several established models estimate R0 and the corresponding effective reproduction number (Rt) at a given time $t$, these models often only take into account epidemiological factors. Thus, the goal of this work is to examine the influence that different sociodemographic factors have on Rt. Based on literature and data availability, different sociodemographic factors spanning population-age distributions, healthcare indicators, and income in relation to poverty measurements were selected as factors of interest. Mobility patterns and restrictions imposed by date factors were also examined at two different time

points to account for temporal Rt changes. Data was analyzed on a state by state basis and distributions and correlations were undertaken in order to identify bias or skew that could impact the models.After performing feature selection on the factors identified, a random forest model and a linear regression model were fitted to the data. Of the sociodemographic factors identified, unemployment rates, median income, and age distributions were found to have the most influence on Rt. Unsurprisingly, restrictions imposed by lockdowns had the single strongest influence on Rt. While this study establishes the role that sociodemographic factors play in Rt, further work to increase the granularity of this analysis this allowing states to perform public health interventions with increased precision is of interest.

Mentor: Tesla La Touche

Keywords: Reproduction Number, Public Health, Sociodemographic, COVID-19 


\section{Correlation between COVID-19, Mental IIIness and Substance Abuse Prevalence}

Robert Joseph, Hamza Qureshi, and Shonnae Frazer

University of Alberta, Brock University, and York University

The aim of this paper is to determine if the experience of the COVID-19 pandemic correlates with worsened mental health and increased substance use among the affected populations. Data sets from the National Addiction \& HIV Data Archive Program (NAHDAP) and Statistics Canada (StatCan) were analyzed, with the aim of determining the trends in mental health and alcohol purchases during the COVID-19 pandemic. Using machine learning model techniques, a strong association between the two was discovered. It was also predicted that similar future situations would yield the same results. Statistical tools within Python, R, and SAS were used to explore the data and analyse it using various techniques., including linear regression. As a result, our findings demonstrate that coronavirus pandemics correlate with increased mental illness and substance use within the affected populations. If its effects are similar to SARS, however, considering that COVID-19 has had a much more widespread impact, significant increases in mental illness and substance abuse can be expected in the months and years to come. The analysis determined that there is a strong correlation between the COVID-19 pandemic, mental illness and alcohol and drug use. Limitations to this conclusion involve the fact that the pandemic is still ongoing and the datasets used were relatively new and small.

Mentor: Sanjay Arumugam

\section{Race, Ethnicity, and COVID-19}

\section{Shivani A. Solanki, and Laura S. Mosquera Pava}

\section{MacEwan University, University of Alberta}

The COVID-19 pandemic has put the world in a state of emergency and affected lives worldwide. Currently, one of the most affected countries by the pandemic is the United State of America. The US is also steadily documenting demographic factors in the confirmed cases and death tolls plaguing the country. As data is being gathered a trend was highlighted in which COVID-19 disproportionately affects minorities. It is important to take a further look into the experiences of minority groups during times of crisis due to the socioeconomic and health inequalities that affect well-being. The following research looks into health inequity and how racial and ethnic minorities' health status and mortality is reflective of the unequal COVID-19 case observations.

\section{Mentor: Hannen Abbas}

Keywords: Race, Ethnicity, Health inequity, Health policy, COVID-19

\section{Reduction of Inmate Population in State Prisons during the COVID-19 Pandemic Based on Advisable Placements for Vulnerable Populations}

Scott Blender, Serene Feng, Regina Liu, and Kelly Ly

Georgia Institute of Technology, University of Connecticut, Mercer University, University of Massachusetts

The COVID-19 pandemic has infected millions worldwide and strict social distancing measures have been enacted to reduce infection rates and contain the spread. However, there has been increased concern regarding the rapid transmission of COVID-19 within densely populated correctional facilities, namely prisons, because they are composed of many atrisk individuals who lack access to adequate healthcare and cannot practice social distancing in confinement. Individuals against the call for a reduction of inmate population have expressed concerns in regards to public safety after inmate releases. Therefore, this study aims to utilize historical mass release and recidivism rate data in order to analyze which populations of inmates can be reduced while also ensuring public safety. We analyzed open-source datasets on prison population, offense, and recidivism rates using the data visualization software Tableau. Our results indicate that releasing inmates over 64 years old and placing them into Geriatric Parole among other recommended placements are proven methods to effectively and safely reduce prison population in a time of emergency. 
Mentor: Jason Steffener

Keywords: COVID-19, decarceration, mass release, recidivism, infection rate

\section{The impact of COVID-19 on illicit drug toxicity deaths in British Columbia - An evaluation of annual coroner reports from 2010-2020}

\section{Sara Emira, Watfa Abou Kaaf, and Atika Chehab \\ McMaster University}

The use of illicit substances has been a growing issue in Canada over the past decade. Despite being a national crisis, certain regions are disproportionately impacted, with B.C. being one of the hardest-hit regions [1]. In recent weeks, numerous articles have reported a significant spike in overdose deaths in B.C. which coincides with the implementation of COVID-19 lockdown measures. In order to assess the validity of these claims, our team used SAS to perform Chi-Square Tests to look for year-to-year trends as well as seasonal trends. The results of these tests support claims of a significant spike in deaths in 2020. Possible causes include high rates of unemployment, depression and social isolation coupled with a reduction in the availability of rehabilitation services. Policy implications include ensuring safe access to substances, helping users maintain a sense of community while being physically distanced, and removing barriers to accessing care despite lockdown measures.

Mentor: Danielle Roy

Keywords: British Columbia, COVID-19, substance use, unnatural deaths

\section{Investigating the impact of twelve socioeconomic factors on COVID-19 death rates for 37 OECD Countries}

Steffani Grondin, Lorne Mallin, Katherine Rink, and Maryum Sayeed

University of British Columbia

The severe acute respiratory syndrome coronavirus 2 (SARS-CoV-2) causing coronavirus disease (COVID-19) has led to over 300,000 deaths in six months since the first known case in 2019. Declared a global pandemic by the World Health Organization in March 2020, COVID-19 has had an unprecedented global impact on social behaviour and national economies. The number of COVID-19 related deaths continues to increase, thus studying socioeconomic factors that could be correlated with COVID-19 death rates could help inform policy and regulation around ending the global pandemic. In this study, we investigate the effect of twelve socioeconomic factors on COVID-19 death rates for 37 countries in the Organisation for Economic Cooperation and Development (OECD). The socioeconomic factors evaluated include poverty rate, poverty gap, income inequality, gross domestic product (GDP), hospital density, and healthcare expenditure as a percentage of GDP. We investigate the correlation between each factor and use our findings to create a linear-log regression model to construct a multivariable function for predicting COVID-19 death rate. We find that the most deterministic factors for predicting COVID-19 death rate when used together in our models are healthcare expenditure, hospital density, GDP, and the overall poverty rate. Although we find no correlation between a country's death rate and their poverty gap or income inequality, we find a negative correlation between death rate and hospital density, and between death rate and poverty rate for seniors specifically. Our data also show that while Western European countries have the highest regional death rates, they have some of the lowest income inequalities, poverty gaps, and poverty rates, indicating that other factors may be correlated to their COVID-19 death rates. Thus, we implore that future studies include more countries and analyze other socioeconomic factors such as population density, substance abuse, and underlying health conditions. Such studies will allow us to make appropriate recommendations to policy makers in order to assist in the efficient distribution of vaccines, as well as developing a more detailed plan for future disease outbreaks.

Mentor: John Spinelli

Keywords: COVID-19, regression modelling, disease outbreak, socioeconomic factors, death rates

The HIV/AIDS Crisis: How Stigma finds its way 


\section{into Policy}

Shanza Jamshed, Amrita Debnath, and Abdullah Aslam

University of Waterloo, McMaster University, Ryerson University

HIV/AIDS is one of the most debilitating public health crises of the last century, made worse by the stigma surrounding what was thought to be a target demographic: men who engage in intercourse with men. While the spread of misinformation has always had consequences, the scale with which repercussions occur is now astronomical. Misconceptions regarding patients with HIV/AIDS has manifested in discriminatory policies in the selection criteria for blood donations. This analysis uses open data extracted from the Public Health Agency of Canada to determine the rates of HIVIAIDS cases by age and sex over time, as well as, whether men who engage in intercourse with men have a higher incidence rate than men who do not. In a 5-year period, there was a significant decline in HIV cases among the gay, bisexual, men who have sex with men (gbMSM) group. This review focuses on addressing the challenges faced by men and women suffering with HIV and current approaches to increasing HIV awareness while simultaneously, decreasing the spread of HIV.

\section{Mentor: Mark Edbed}

Keywords: HIV, sexuality, social stigma, transmission, social media

\section{Analyzing the Effect of Social Distancing Policy Implementation on Decreasing COVID-19 Infection Rates}

Sophia Lu, Jen Guo, Sathvika Mudigonda, and Vivian Huynh

\section{University of Calgary}

The novel coronavirus (COVID-19) is a highly contagious disease that has impacted the health of individuals worldwide. Currently, Canadian interventions have focused on physical distancing measures to contain the spread of the virus. This study aims to evaluate the extent to which social distancing policies result in effective disease containment in four Canadian provinces: Alberta, British Columbia, Quebec, and Ontario. Data of the confirmed COVID-19 cases worldwide and the dates of social distancing policy implementations were obtained from the Institute for Health Metrics and Evaluation open-source database. The dataset was compiled from public government websites and the Johns Hopkins University data repository. The cumulative confirmed infections were plotted as a function of time using $\mathrm{R}$ software and fitted using polynomial regression. Second or third order regression models were used with a $95 \%$ confidence interval. Data analysis showed that prior to the introduction of social distancing measures, all four provinces displayed an increasing positive slope, suggesting a nonlinear increase in new daily infections over time. However, after the initial implementation of social distancing policies, all four provinces displayed decreasing slope values approaching a plateau, indicating that social distancing policy implementations were beneficial in containing the spread of COVID-19. Furthermore, British Columbia had the most drastic decrease in infections compared to the other three provinces after policy implementation, despite having a higher number of initial cases. The results suggest that provinces that implement social distancing measures during the steady state of the infection spread may fare better in flattening the disease progression curve. Because the impacts of social distancing policies on the spread of COVID-19 have not been thoroughly studied in Canadian populations, this finding has pertinent contribution to forming a plan of action for future waves and outbreaks of COVID-19 and other similar viruses.

Mentor: Sanjai Arumugam Jaganmohan

Keywords: COVID-19, corona virus, COVID-19 infection rates, social distancing, quarantine

\section{Effects of Political Leaders on Public Opinion Regarding COVID-19}

Stuart Martin, Jenney Ren, Riju Sikdar, and Joyce Li University of Guelph, University of Toronto

As the COVID-19 pandemic continues to ravage the globe, many political leaders are encouraging or enforcing social distancing to limit the spread of the virus. In the United States, social distancing is largely voluntary. As such, public 
opinion on the necessity of self-isolation directly affects the number of COVID-19 cases in the area. Our study uses sentiment analysis on state governors' Tweets to determine whether communications vary between Republican and Democrat governors. We used open resources such as Twitter Developer Labs and Python 3, to collect all governor Tweets throughout the pandemic and perform automated sentiment analysis. We found that compared to Democrat governors, Republican governors had a higher proportion of positive and neutral posts and a lower proportion of negative posts. On average, posts from both parties were equally subjective and Republican posts were more positive. Given prior literature on the effects of communications from political leaders on the public, we conclude that a unified political stance on social distancing would increase the effectiveness of social distancing orders.

Mentor: Nayaar Islam

Keywords: COVID-19, politics, sentiment analysis, United States

\section{An Exploratory Analysis of the Impact of the Legalization of Medical Assistance in Dying (MAID) in Canada on Life Expectancy, Healthcare Expenditures, and Non-medical Suicide Rates}

\section{Safana Nasir}

University of Toronto

Medical assistance in dying (MAID) is a procedure for a patient to safely and intentionally end their life if they meet strict criteria. In June 2016, great debates were sparked as MAID was legalized in Canada. The objective of this paper is to explore how the legalization of MAID has impacted the outcomes of life expectancy, healthcare expenditures and non-medical suicide rates in Canada. Implications of the statistical analysis enable a critical and objective understanding of the claims made in the media and evaluate the extent to which MAID complies with law, ethics and patient care. Data was retrieved from portals, including Statistics Canada, Macrotrends and the National Health Expenditure Database. The data analysis compared three main outcomes before and after the legalization of MAID: life expectancy, healthcare costs, and suicide rates. Regression analysis was performed using linear models to compare the actual data of the outcomes and the projections produced by the regression lines. The results indicated that following legalization in 2016, life expectancy slightly decreased, healthcare expenditure had no meaningful change, and non-medical suicide rates decreased. However, no causal relationships or definite conclusions were rendered due to limited data. For future research, the information in the article should be revisited as available data increases over the years and legalization becomes widespread. For further investigation, links between MAID and other factors, such as palliative care and depression rates, can be researched.

\section{Mentor: Daiva Nielsen}

Keywords: medical assistance in dying, medically assisted death, MAID, MAID legalization

\section{An Investigation into the Long-term Efficacy of Various Policies Regarding COVID-19}

Sai Panidarapu, Evan Maloney, Remi Hou, and Shubh Patel

University of Alberta, University of Calgary

The overwhelming cases of COVID-19 in the past few months across the world have in many ways pushed the limits of a country's ability to deal with such a large array of issues in a short period of time. Policies ranging from being centered around international affairs to even managing public events have been dramatically altered. The aim of this study was to see which of these altered policies had the greatest effects in slowing down the death rates of their respective countries or whether some unknown confounding variable is the major contributor. To achieve this, several countries were selected to be analyzed over the period from March 10, 2020 to April 10, 2020 - when most countries have responded to the pandemic. Countries were chosen such that they had as many differences as possible over the course of $\mathbf{3 0}$ days, allowing for a better comparison between specific policies. For example, if countries had very similar policies, there would not be much to compare. Subsequently, total deaths versus time for each country were graphed and a trend-line was produced for which a function was developed. The derivatives of these 
functions were manually calculated to achieve a function for the death rate, which was then graphed in $\mathrm{R}$ studio. In addition, using a rubric that was developed in regards to how strictly each policy was implemented, countries were assigned scores. The average score over the time period was calculated and this was compared to the death rate graphs in order to see if there was any correlation between the severity these policies were implemented and the trend in death rates experienced for each country.

\section{Mentor: Matineh Panah}

Keywords: COVID-19, policies, effectiveness, Europe, pandemic, coronavirus, death rate

\section{Public Health Policy and its Effect on the Reproductive Number for COVID-19}

Srinija Palaparty, Shriya Parikh, Tarjoth Khaira, and Tarun Sharma

\section{University of Waterloo}

Late in the year of 2019, Wuhan, China discovered the existence of a lethal and unknown virus known as COVID-19 within their community's population. COVID-19, also known as SARS-CoV-2, is an acute respiratory illness that attacks the lungs, and is transmitted through respiratory droplets. Large, heavily populated countries, such as the United States of America, were taken aback by the repercussions and advanced spread of the COVID-19 virus. Data was used from the open-source, Our World Data and processed in MATLAB where the code package, fitVirusCOVID19 from MathWorks file exchange was used to generate $\mathrm{R}$ numbers and graph them for the countries Canada, the United States, South Korea and New Zealand. It was observed from these results that countries with previous experience in combating a pandemic, such as SARS or MERS, were successful in containing the spread of the coronavirus. By having a global plan, such as the Pandemic Preparedness Act, which functions as a baseline for an effective response, effects of a global pandemic can be mitigated. The act consists of three policies. The first policy will require countries to report the outbreak of a virus as soon as it becomes an epidemic. The second policy will ensure that countries will take a maximum of two weeks between the hundredth reported case and the enforcement of a nationwide lock down. The third policy will have countries conducting mass testing so that infected individuals are able to be diagnosed and isolated. An act developed to prevent the spread of future diseases will prove to be beneficial as it will limit the invasion of the disease while not putting the health of citizens at risk.

Mentor: Mark Edbed

Keywords: COVID-19, coronavirus, SARS, R number

\section{Investigating Intrauterine Transmission of COVID-19}

Smeet Solanki, Sudhanva Suvarna, Feny Pandya, and Venezia Prochilo

McMaster University

In December 2019, a novel coronavirus (COVID-19) originating from Wuhan, China began to spread worldwide at an exponential rate, eventually leading to a global pandemic. This paper investigates the possibility of intrauterine transmission of COVID-19 by compiling data from recent studies on COVID-19 test results for pregnant women and neonates. Although there is no direct evidence for intrauterine transmission, 13 neonates tested positive for the virus, therefore this possibility cannot be ruled out at this time. These neonates faced adverse outcomes following birth, however it is unknown if these outcomes were present due to infection. Thus, future studies are required to support these assertions with stronger evidence.

Mentor: Dan Gillis

Keywords: COVID-19, Coronavirus, Neonate, Pregnancy, Intrauterine

\section{Correlation between days since first case of nCOVID-19 and case fatality rate in Southern European Countries}

\section{Stanley Z. Hua \\ University of Toronto}

In the midst of a pandemic, there is great demand for resources and information. Yet, as focus is placed on 
"flattening the curve", the question needs to be asked: 'How well are we taking care of those infected?' A possible measure for this is the case fatality rate, which is defined as the proportion of infected who have died due to disease.[1] Through this paper, we hope to shed light on the question raised by uncovering potential relationships in up-to-date nCOVID-19 data. To address this, exploratory data analysis and statistical analysis on data retrieved from the $\mathrm{R}$ package COVID19[2] and The World Data Bank[3] were done using R. Results imply a moderately strong correlation between the number of days since the first case and case fatality rate for respective countries in Southern Europe (with 1000 cases or above). We believe that this finding has potential in supplementing future studies that hope to analyze actions the governments have taken and will take to improve public health systems and policies in treating those infected. As more information is available every day, it is recommended that future studies, hoping to study case (or infection) fatality rates between countries, find and implement reliable estimates for the true number of nCOVID-19 cases in each country.

Mentor: HuyThong Bui

Keywords: COVD-19, case fatality, southern europe

\section{How Lifestyle Factors and Health Conditions in Seniors Correlates to COVID-19 Case Outcomes in Canadian Provinces}

\section{Theodore Leung and Ellison Tong}

\section{Simon Fraser University}

Canada has seen large outbreaks in COVID-19 deaths with its seniors (65 years or older) that resided in longterm care facilities and nursing homes. The extent of these occurrences are so wide-spread that nearly half of COVID-19 deaths are connected to these types of institutions. Given how vulnerable this age group is towards COVID-19, more analysis must be done in order to minimize the fatality rates for Canadian seniors. This paper aims to further understanding of COVID-19 induced deaths in seniors by analyzing the correlations between COVID-19 case outcomes (confirmed cases, recovery rates, deceased, etc.) and different health factors among seniors. The data used in this paper are from the Government of Canada's Public Health Info Base and Statistics Canada. Due to the large number of variables considered in this study, heat maps were used to detect high levels of correlation. Linear regression between highly correlating variables was computed and plotted to visualise the spread of data. It was found that seniors who have chronic obstructive pulmonary disorder (COPD), fair or poor perceived health, high blood pressure and obesity were associated with having moderately fewer positive COVID-19 test results. These results suggest that seniors with these conditions or the people in their social networks, or both are taking more precautions with COVID-19. Another possible explanation is that these seniors have certain lifestyles that lead to less contact with COVID-19. These findings suggests that more awareness of hygienic habits is needed for seniors that don't fit into these low-risk groups and for those in their social networks. Future research may focus on investigating the specific reasons as to why seniors with COPD, fair or poor perceived health, high blood pressure or obesity are associated with lower positive COVID-19 cases.

Mentor: Deepak Reddy Mandadi

Keywords: COVID-19, seniors, comorbidity, deaths, lifestyle

\section{Foot traffic in different Sectors across Canadian Provinces and its impact on COVID-19 Transmission during Self-isolation}

Tony Liu, Shawn Zhang, Natalie Chow, and Jia Hao Chen

\section{University of Toronto, Western University}

The Canadian government's shutdown of the country in response to the COVID-19 pandemic involved social distancing policies in almost all areas. From the correlation between foot traffic in different sectors (i.e. parks, residential areas, workplaces, transit, etc.) and new COVID-19 cases, we hoped to better understand the areas in which social distancing is more or less effective at minimizing transmission. Using factor analysis, clusters were extracted from Google's Community Mobility dataset. Foot traffic data from these clusters were graphed against the change in daily COVID-19 cases from Health Canada's COVID-19 infections dataset and fitted to a linear regression model. The results showed no 
significant correlation between change in foot traffic across the different clusters and COVID-19 transmission. However, parks had weak correlation with new COVID-19 cases across provinces, suggesting earlier re-opening policies are possible. Within privacy restrictions, we recommend further data collection on foot traffic during pandemic situations before implementing change in the location and timing of social distancing and re-opening policies.

\section{Mentor: Kamal Ben}

Keywords: COVID-19, transmission, sectors, mobility, Canada, provinces

\section{An Exploratory Analysis of Public Sentiment Throughout the Covid-19 Pandemic}

\section{Ty Werbicki, Harry Wilton-Clark, and Tyler Sawatzky}

University of Calgary

During the Covid-19 pandemic, current policies and resources are being directed towards primary health concerns such as protecting vulnerable health populations and flattening the viral curve. While focusing on these initiatives is mandatory to maximize public health, this leaves room for secondary concerns to slip through the cracks, such as adverse mental health effects. Current models predict an additional 10,000 annual suicides due to COVID-19-related factors. Thus, the timely and upstream identification of how COVID-19 and its policies are affecting population mental health is imperative. We used a Python library to scrape and analyze a high volume of tweets, providing each tweet with a positive or negative sentiment score. Sentiment scores were compared across Texas and California, two states with opposing COVID-19 policies. Our data identified a clear deterioration in public sentiment which follows major developments in the global COVD-19 narrative. Additionally, we found that public sentiment was significantly reduced in California under strict policy compared to public sentiment in Texas under looser policy. These findings validate existing models which predict an impending mental health crisis. Policymakers should use these preliminary findings as early evidence to support implementation of upstream mental health policy, and to elucidate the clear link between quarantine policy severity and adverse public mental health.

Mentor: Lydia Odilinye

Keywords: COVID-19, Depression, Mental, Health, Policy

\section{An Exploratory Analysis for the possibility of Utilizing technology to prevent Type II Diabetes}

\section{Tiffany Wai}

\section{University of British Columbia}

This paper aims to determine the possibility of utilizing technology to prevent Type II diabetes. Type II diabetes was chosen for the investigation due to its general nature across Canada and the huge medical expenses the Canadian Government has to put every year. Prevention of Type II diabetes was not a focus on health policies and was carried on a small-scale. This paper would be analyzing datasets from The Canadian Health Measures Survey (CHMS), research done by Wang et al., and Gabbiadini et al. The paper would put a focus on evaluating the effectiveness of diet tracking and physical activity tracking when achieving a certain goal. The survey done by current users determined the effectiveness of the current apps in achieving goals. Most people found it effective to use apps in doing diet and physical activity tracking, with more people agreeing that efficiency could be maximized when both apps are used. Pokémon Go was used as a type of virtual game that is analyzed due to the realvirtual interaction between the user and character. Results showed that Pokemon Go could encourage people to continue engaging in physical activity like walking more frequently and running. Analysis proves that this kind of virtual game could attract a group of more ambitious users to engage in physical activity. With the analysis carried out, it has provided a foundation for developing an app that could combine both diet and physical activity tracking. The app could also have a virtual environment so that data collected could be transformed as an award for the virtual character, and users of the app could compete with each other, providing more addiction for them to continue using the app. Users of the app could enjoy the fun of maintaining a healthy lifestyle at the same time preventing Type II diabetes.

Mentor: Dennis McCormac 
Keywords: diabetes, physical activity tracking, diet tracking

\section{An Exploratory Analysis on the Effect of Tree Density on Fine Particulate Matter Levels in New York City}

\section{Waleed Hussain, Sarisha Philip, Vivek Philips, and Imran Uddin}

\section{University of Toronto, McMaster University}

A commonly overlooked factor in public health is air quality, especially in major urban centers where there are numerous sources of air pollution. Evidence shows that poor air quality can lead to various downstream health complications such including respiratory, cardiovascular and psychological complications[1]. A major air pollutant of note is particulate matter smaller than 2.5 microns (PM2.5). These particles are small enough to pass into the body and cause both short term and long term health problems[1]. Studies have shown that trees can reduce PM2.5 in the surrounding area, and thus it is logical to suggest that there may be a greater effect with additional trees. This study aimed to determine whether higher tree densities in a given area could reduce PM2.5 concentrations[2]. Using ArcGIS Pro and R Data, both air quality (NYC Community Air Survey) and tree density (2015 Street Tree Census) were spatially related and analyzed to determine a potential relationship. Road data was also used to control for known contributors to PM2.5. The analysis seems to provide evidence for an inverse relationship between tree density and PM2.5 concentration. Furthermore, the analysis provided evidence for a positive correlation between PM2.5 concentrations and road width. With more research, urban planners can leverage better street and green-space design to maximize air quality and health consequences.

Mentor: Gloria Lim

Keywords: air pollution, trees, urban planning, public health
Wanying Ye, Saim Imran, Yuan Bie, and Hanyi Weng

University of Toronto

HIV is a public health concern worldwide because no cure or vaccine exists and the only treatment available is ART. However, because many individuals remain untested, not all HIV-positive individuals receive treatment. Therefore, WHO and UNAIDS created the $90-90-90$ target to have $90 \%$ of HIV-positive patients tested and on treatment in order to stymie the epidemic. Canada, amongst countries striving towards this target, has emphasized education of vulnerable populations to increase testing rate. The aim of this paper is to gain better insight into reasons why people might avoid HIV testing, especially with regards to misinformation and attitudes towards the efficacy of treatment between age groups and between provinces. Analysis of a 2018 PHAC survey on STBBI data was performed to find trends for misinformation, stigma, and willingness to get tested. Data was cleaned, sorted, and displayed into bar graphs, pictograms, pie charts, and maps using Rstudio. Results show that 16 to 24-year-olds were most misinformed and at risk of infection, while participants aged 16 to 44 were more comfortable seeking HIV information online than from their doctors. Quebec displayed lack of awareness for needle sharing leading to HIV infection while Saskatchewan demonstrated a high indication of inaccessibilty to healthcare as a primary reason for not getting tested. Creating policies to target certain age groups, such as increasing accessibility of accurate HIV information online and removing stigma would help Canada reach the 90-90-90 target. Moreover, different platforms for correcting misinformation can be tailored to the characteristics of different age groups as there exists differences in information seeking preference.

Mentor: Vidhi Patel

Keywords: HIV, misinformation, policy, testing, 90-90-90 target

\section{Association of an Area's Income Level with How It Responds to Health Crises}

Xinyun Ren, Chenchen Tang, Yuxin Jia, and Haocheng Chang 


\section{University of Waterloo}

In light of the outbreak of COVID-19, populations with different income levels responded differently in personal care actions such as buying masks and seeking medical treatments, working remotely from home or becoming unemployed. This phenomenon has arisen the question of whether household income levels have a direct correlation to region's infection and unemployment rates. Those with higher incomes typically achieve higher levels of education, and therefore take more precautions over personal health and care, leading to lower infection rates. Data of median household income, infection rate, and unemployment rate in provinces of Canada and states of America are collected and analyzed through various models. It is found that there is a positive relationship between household income level and infection rate, and there is no clear relationship between household income level and unemployment rate. The various causes behind these relationships are also analyzed.

Mentor: HuyThong Bui

\section{An In-Depth Study for the Economic Impact of COVID-19 on Oil \& Gas and Mining Industries}

Xiao Wang, Tong Wang, Freya Zhang, and Lily Zhang

\section{McGill University}

Having fulfilled all required epidemiological criteria of a pandemic, coronavirus disease 2019 (COVID-19) has spread rapidly and infected more than 6 million people globally; to most countries, it has triggered grave concerns re garding their healthcare systems, politics, and economics [1]. Canada, as a developed country with a health system ranked among world's best, yet has more than 90,000 confirmed cases of COVID-19 currently and attempted to implement various measures to contain economic fallout in its major industries. Herein, the economic impacts of COVID-19 on Canada's oil \& gas and mining industries were explored and discussed in a descriptive manner, with explanations rationalized and unveiled. The COVID-19 data used were collected from the Public Health Agency of Canada [2], whereas the stock prices data were obtained from Yahoo Finance [3-14]. The stock prices for selected companies were plotted against the total confirmed cases of COVID-19 in Canada between March 11th and May 9th, to which a set of segmented linear regression models were applied to evaluate key changes. Studentized tests were used to validate the regression models, and normalization of data were introduced additionally to depict the overall finding. With these statistical and analytical tools, it was clearly found that the segmented regression models were indeed accurate predictors with a few exceptions - an inflection point where key changes might have taken place occurred. Conclusively, several reasonable explanations regarding these changes were proposed, including government actions, Russia-Saudi Arabia oil price war, reduced transportation, and varying prices of gold and oil. In the future, the influence of the stock markets in other countries rather than Canada can be incorporated and a larger data set may be selected to provide a more complete and persuasive analysis. In brief, this study has proposed meaningful suggestions and employed imitable analytical methods which can engender a far-reaching effect on the future studies.

Mentor: Mohammad El-Hajj

Keywords: COVID-19, economic fallout, stock market, segmented linear regressions, inflection point

\section{Corona-virus and its Impact: A Case of United States}

Xingzuo Zhou, Qi Shu, Xingyou Zhou, and Yixuan Fan

University College London, McMaster University, Canada, University of Toronto, Queen's University, Canada

The paper outlines a framework to analyze impacts of behaviours and policies on COVID-19 transmission in United States. The analysis illustrates the significance of government interventions as tools in delaying virus transmission, and how the speed of that transmission negatively influences the financial market according to key indices of US stock market: Dow Jones Industrial Average and Dow Jones US Health Care Index.

Mentor: Vidhi Patel

Keywords: COVID-19 outbreak, policy, behaviour, data 
science, OLS regression

\section{A Novel Analysis of the Effects of Alcohol Consumption on Cannabis Usage in Youth}

Yudhvir Bhatti, Hertek Gill, and Aditya Prashar

University of Ottawa

Mental health has become a growing concern and unfortunately youth who deal with stress and anxiety may end up resorting to unhealthy behaviours such as cannabis consumption for relief. Furthermore, alcohol consumption within the household has proven to be a leading cause of stress within adolescents. Moreover, with the recent legalization of cannabis in Canada, the drug is more readily available and its increased consumption in youth is not fully understood. This paper aims to determine whether there is a correlation between household alcohol consumption and usage of cannabis among youth. Data from European nations was used as a model to determine the correlation between the consumption of these two substances. Data from various public access databases was analyzed using excel and python 3. Linear regression models, using data for alcohol consumption per capita and cannabis usage among youth showed that in nations with increased alcohol consumption cannabis usage among youth were also higher on average. By studying the relationship between alcohol consumption and cannabis usage in European countries with cannabis decriminalization, showed that legalization did not result in an increase in consumption. This shows that increased access to the drug was not as large of a factor in youth consumption as initially predicted.

Mentor: Tesla La Touche

Keywords: Cannabis, Alcohol Consumption, Decriminalization, Youth

\section{Relationship between people' attitudes towards COVID-19 and its alleviation}

\section{Yunqing Fei and Zixuan Deng}

University of Waterloo, Wilfrid Laurier University

Up to May 31, 2020, COVID-19 has been a raging pandemic for almost three months[1]. Despite the imposed policies, the growth is still continuing in some regions. This triggers us to come up with a hypothesis that the sentiment will affect people's behavior, which, in turn, will affect how efficiently the policies are implemented. If the relationship exists, guidance of public opinion might also be a way to control the outbreaks. Tweeter, as a popular media where people share their thoughts through, is a good source of sentiment data. We collect posts in the US from March 29 to April 30 and do a sentiment analysis by date. Then, we compare this result with the number of new cases in the US and find out that the hypotheses should be rejected based on ANOVA test.

Mentor: Priyanka Gogna

Keywords: COVID-19, Corona Virus, Twitter Sentiment Analysis

\section{COVID-19 in Canada - Prediction on Canadian Recovery Rate}

Yunkun Yang and Jia Xin Li

University of Waterloo

Coronavirus disease (COVID-19) has been a widespread lifethreatening disease in 2020. Under Canadian government's active interventions and the Quarantine Act, the pandemic is increasingly under control, but there is still many people suffering from the symptoms of the disease. We want to know when these people can be fully cured and when the Coronavirus will no longer be a huge public health concern. We used the data from the Government of Canada and constructed a logistic regression model to predict when Canada can recover from the disease.

Mentor: Jenny Bicong Ge

Keywords: COVID-19; Data Analysis; Logistic Regression

\section{Quantifying Time Difference Between Mobility Data and COVID-19 Cases}

\section{Zejia Chen, Joan Lee, Russell Leong, Chelsea Zhang McMaster University}

Since the outbreak of COVID-19, there have been a myriad 
of testing policies and approaches to manage the pandemic that have been employed by different countries. However, these policies have come with varying degrees of success, thus impacting the time lag between infection and diagnosis of COVID-19. This paper aims to determine the time lag between infection and diagnosis of COVID-19 and to investigate how this is impacted by governmental policies. Cross-correlation between the mobility of citizens and COVID-19 cases from Brazil, Canada, Italy, Japan, South Korea and the United States was conducted, followed by a qualitative review of policies implemented by each government. Through these methods, it was determined that there is a Pearson's correlation of 0.865 between time lag and cumulative COVID-19 deaths. This demonstrates that increased time lag was found to correlate with increased mortality, indicating that decreased regulation and control by the government may be quantified using this value. We hope that understanding the COVID-19 time lag between infection and diagnosis could aid in mitigating the pandemic by forecasting spikes in COVID-19 cases. This can have epidemiological implications, as it provides hospitals, financial markets, and governments with advance warning for impending waves of COVID-19 cases.

Mentor: Wanhua Su 